$\mathcal{G}_{\text {http://dx.doi.org/10.3765/sp.8.11 }}^{\text {Semantics \& Pragmatics Volume 8, Article 11: 1-55, } 2015}$

\title{
Investigating the distribution of some (but not all) implicatures using corpora and web-based methods *
}

\author{
Judith Degen \\ Stanford University
}

Submitted 2014-09-10 / First decision 2014-11-26 / Revision received 2015-01-06 / Accepted 2015-01-11 / Published 2015-05-15

\begin{abstract}
A prevalent, but to date untested, assumption about lexicalized scalar implicatures such as those from some to not all, is that they fall into the class of GCIs and as such, constitute a homogeneous class of highly regularized and context-independent implicatures. This paper reports a test of this assumption in which linguistically untrained participants' implicature strength judgments were collected for naturally occurring utterances containing the word some in a large-scale corpus-based web study. The results indicate that implicature strength is highly variable and systematically dependent on features of the linguistic context such as the partitive, determiner strength, and discourse accessibility. These results call into question the GCI status of scalar implicatures from some to not all and demonstrate the usefulness of corpora and web-based methods for challenging received wisdom, enriching the empirical landscape, and informing theory in pragmatics.
\end{abstract}

Keywords: scalar implicature, GCI, corpora, experimental pragmatics

* This paper has benefited greatly from discussion with many wonderful colleagues, including Anton Benz, Michael Franke, Polly Jacobson, Florian Jaeger, and Mike Tanenhaus, as well as members of the Tanenhaus lab and the ZAS Semantikzirkel, to whom I presented parts of this work. I'm very grateful to Greg Carlson, Christine Gunlogson, Dan Lassiter, Roger Levy, Judith Tonhauser, students in the Fall 2014 UCSD Bayesian and Game-Theoretic Pragmatics course, Paul Elbourne, and three anonymous reviewers for reading earlier drafts of this paper and providing helpful comments and discussion. Remaining errors are mine. This work was partially supported by NIH grant HD 27206 awarded to Mike Tanenhaus and NSF CAREER grant BCS-0845059 awarded to T. Florian Jaeger.

(C) 2015

This is an open-access article distributed under the terms of a Creative Commons Attribution License (http://creativecommons.org/licenses/by/3.o/). 


\section{Introduction}

Ever since Logic and Conversation (Grice 1975), scalar implicature has been treated as an instance of Generalized Conversational Implicature (GCI). That is, scalar implicatures are assumed to arise as a matter of default, independently of context, though they may be canceled if not licensed by the context. This sets them apart from Particularized Conversational Implicatures (PCI), which rely heavily on the context of utterance. The GCI status of scalar implicatures is a fundamental assumption both for theories of the conditions under which scalar implicatures arise (e.g., Gazdar 1979, Horn 1984, Levinson 2000) as well as for theories of how scalar implicatures are processed (e.g., Levinson 2000).

Unfortunately, the data to support the categorization of scalar implicatures as GCIs - or indeed, the categorization of any sort of implicature as a GCI or PCI - have thus far consisted entirely of linguists' intuitions, typically just the authors', using a handful of examples. This was perfectly justified at the time that conversational implicatures were first investigated, when the tools to collect large quantities of regular language users' judgments across different contexts were not available. However, the small number of experimental participants - the author(s) - and experimental items - the handful selected by the author(s) - can introduce bias and call into question the generalizability of theories that are developed by this method (Gibson, Piantadosi \& Fedorenko 2011).

Fortunately, researchers now have access to large-scale corpora of spontaneous speech as well as the ability to collect judgments from a diverse population and large number of experimental participants over the web. That is, we now have at our disposal the means to empirically test the validity of claims concerning the context-independence and defaultness of various types of conversational implicatures.

This paper takes a modest first step in this direction by testing the assumption - which I will refer to as the Homogeneity Assumption - that scalar implicatures from some to not all constitute a homogeneous, contextindependent type of implicature that falls into the class of GCIs. This will be achieved by conducting a large-scale web-based study in which regular language users' interpretations of utterances containing some, extracted from a corpus of spontaneous speech, are collected.

In the rest of this section, I discuss what is at stake in testing the Homogeneity Assumption. In Section 2 I report the study, which aims at testing two 
Distribution of scalar implicatures

aspects of the Homogeneity Assumption: (a) whether there is variation in the strength with which scalar implicatures from some to not all arise, and (b) whether there is systematic context-dependence in this variation. In Section 3 I discuss the implications of the results for the status of scalar implicatures as GCIs, and for theories that rely on scalar implicatures constituting a homogeneous class of implicatures.

\subsection{What is at stake}

Most linguistic and psychological processing theories of scalar implicatures make the Homogeneity Assumption to some extent (Gazdar 1979, Horn 1984, Levinson 2000, Huang \& Snedeker 2009). ${ }^{1}$ It is thus crucial to be explicit about what exactly the Homogeneity Assumption entails and what the consequences would be, should it be overturned. This is what this section is devoted to.

\subsubsection{The Homogeneity Assumption and the GCI-PCI distinction}

The Homogeneity Assumption can be stated as in (1) and includes the subassumptions in (1a) and (1b).

(1) The Homogeneity Assumption

Lexicalized scalar implicatures constitute a homogeneous class of implicatures.

a. STRENGTH INVARIANCE: Implicature strength is not variable.

b. CONTEXT INDEPENDENCE: Implicature strength is not systematically dependent on context.

I will demonstrate how the general assumption follows from the GCI-PCI distinction and elaborate on each of the sub-assumptions in turn.

The GCI-PCI distinction. Consider (3) as an answer to (2a). Dan can be taken to mean that not all of the students failed, and in addition that the exam

1 Most of the processing literature has been careful to remain non-committal about the status of scalar implicatures as GCIs, or has argued against the usefulness of GCI as a psychological term (Breheny, Katsos \& Williams 2006, Breheny, Ferguson \& Katsos 2013). However, this literature relies on scalar implicature processing being comparable across different experiments, and thus, across different linguistic and discourse contexts (e.g., Bott \& Noveck 2004, Huang \& Snedeker 2009, Grodner, Klein, et al. 2010, Bott, Bailey \& Grodner 2012). It is in this sense that one of the aspects of the GCI claim is implicitly endorsed. 
was hard. In contrast, consider Dan's utterance as a response to (2b): In this case, the scalar implicature that not all of the students failed still goes through, but Dan can no longer be taken to implicate (4a). However, now he can be taken to implicate that the teacher did not do a good job, which was not an available implicature in (2a). These kinds of observations of scalar implicatures seemingly arising independently of context have contributed to their analysis as GCIs in contrast to the more context-dependent PCIs in (4).

(2) a. Masha: Was the exam hard?

b. Masha: Did the teacher do a good job?

(3) Dan: Some of the students failed. $m$ Some, but not all, of the students failed. (GCI)

(4) a. $\leadsto$ The exam was hard. (PCI)

b. $\leadsto$ The teacher did not do a good job. (PCI)

Grice characterizes the distinction between the two types of inferences as follows: PCIs are carried by "saying that $p$ on a particular occasion in virtue of special features of the context, cases in which there is no room for the idea that an implicature of this sort is normally carried by saying that p" (Grice 1975, p. 56, emphasis in the original). In contrast, of GCIs he says "the use of a certain form of words in an utterance would normally (in the ABSENCE of special circumstances) carry such-and-such an implicature or type of implicature" (Grice 1975, p. 56, emphasis in the original).

There is agreement in the literature that not all scales are created equal that is, some scales are more readily involved in the generation of scalar implicatures than others. This is captured in the distinction between lexicalized and ad hoc scales (Hirschberg 1985, Horn 1989, Matsumoto 1995). Lexicalized scales are such that whenever the weaker element from the scale is observed, the stronger one functions as an alternative. Scales that have been proposed to be lexicalized are those made up of quantifiers like $\langle$ all, some $\rangle$, sentential connectives like $\langle$ and, or $\rangle$, modals like $\langle$ must, can $\rangle$, or numerals like $\langle$ three, $t w o\rangle$. In contrast, ad hoc scales require more context to become functional. Hirschberg (1985) has noted that any items that constitute a partially ordered set in which one item can be determined to be higher than another one can function as a scale. For example, the scale $\langle$ send, write $\rangle$ contains different stages an email may be in, where sending it follows writing it. However, for this scale to become functional, the context needs to be such that sending the email is a salient competitor to writing it. It is probably not 
the case that most utterances of I wrote the email compete with I sent the email.

The seeming context independence of lexicalized scales and the context dependence of ad hoc scales has been used to categorize lexicalized scalar implicatures as GCIs and ad hoc scalar implicatures as PCIs. Since the Homogeneity Assumption is formulated only for GCIs, not for PCIs, it should hold for lexicalized scales, but there is no expectation that it should hold for ad hoc scales. The most discussed and unambiguously agreed-upon case of a lexicalized scale is the $\langle$ all, some $\rangle$ scale. Thus, if the Homogeneity Assumption holds at all, it should hold for implicatures from some to not all.

In what way then are some-not-all implicatures assumed to constitute a homogeneous class of implicatures? Precisely in the way specified by the GCI-PCI distinction: GCIs set themselves apart from PCIs in that they usually arise (captured by sub-assumption (1a): STRENGTH INVARIANCE) and survive context shifts (captured by sub-assumption (1b): CONTEXT INDEPENDENCE).

A test of the Homogeneity Assumption is crucial not only to theories of the conditions under which scalar implicatures arise, but also to theories of how they are processed. ${ }^{2}$ Both of the main rival processing theories of scalar implicature - the Default model (Levinson 2000) and the Literal-First hypothesis (Huang \& Snedeker 2009) - make the Homogeneity Assumption, though the respective status of the assumption in the theories differs. For Levinson it is a core assumption of the theory; for the Literal-First hypothesis it is a background assumption that allows processing delays in computing scalar implicatures to be interpreted as evidence for a processing distinction between semantics and pragmatics, and in particular for a privileged position of computing literal content over computing pragmatic implicatures. Thus, while the Homogeneity Assumption is necessary for the interpretation of delayed implicature processing results as support for the Literal-First hypothesis, overturning it would not call into question the theory itself, though it would call into question the testability of the theory. I defer a fuller discussion of the Literal-First hypothesis to the general discussion in Section 3, and focus here on the consequences for the Default model.

Levinson (2000) postulates default, cognitively cost-free GCIs as a solution to what he calls the articulatory bottleneck problem: There is a significant articulatory bottleneck in the rate of information that can be transmitted via human speech (estimated as out-of-context phoneme information). In addition, he assumes that integrating contextual information to derive com-

2 These are not unrelated in principle, but often are in practice. 
plex pragmatic inferences is cognitively effortful. ${ }^{3}$ Nevertheless, linguistic communication proceeds at a miraculous speed. Thus, the communicative system must have evolved a solution that allows for rapid communication through a very limited channel. The solution, according to Levinson, is to make inference cheap for hearers on average - and the best way to do this is to allow for highly regularized inferences (GCIs) to be derived at no cost, thus balancing out the cost of deriving difficult contextual inferences (PCIs). This balance of costs would allow communication to proceed at the rapid rate at which it does. ${ }^{4}$

Thus, both STRENGTH INVARIANCE and CONTEXT INDEPENDENCE are crucial to the Default model: if scalar implicatures from some to not all are in fact much weaker, less regularized, and more context-dependent than Levinson assumes, this would mean that scalar implicature processing involves much more cognitive cost due to implicature cancellation and integration of contextual information than previously assumed. If this result generalizes to other lexicalized scalar implicatures, this would mean that cognitive cost-freeness of GCIs does not clearly constitute a solution to the articulatory bottleneck problem.

In the following I discuss the sub-assumptions in more detail and clarify the empirical predictions that the Homogeneity Assumption makes.

3 This assumption, while intuitive and common in the linguistic literature, is unfortunately wrong. There is much evidence from the psycholinguistic literature that suggests that hearers can very rapidly integrate information from multiple contextual cues. For example, the visual context has immediate effects on whether a prepositional phrase is interpreted as a destination or as modifying a definite NP (Tanenhaus et al. 1995); an object's affordances may immediately disqualify it as a potential referent (Chambers, Tanenhaus \& Magnuson 2004); whether a particular piece of information is in common or privileged ground can immediately affect the interpretation of definite NPs with prenominal scalar adjectives (Sedivy et al. 1999, Heller, Grodner \& Tanenhaus 2008); and whether a speaker is deemed reliable with respect to the degree with which he over-informs can have rapid effects on contrastive inferences (Grodner \& Sedivy 2011). Thus, processing contextual information may not in fact be costly, and so neither may processing PCIs which crucially depend on processing of contextual information. This assumption of Levinson's is thus highly questionable, but I will not discuss it further here.

4 Note that there are no actual estimates of how rapidly communication should proceed under different assumptions about the cost of various inferences. That is, intuitions about the rate at which communication should proceed for different cost distributions, are in effect no more than that - intuitions. 
Distribution of scalar implicatures

STRENGTH INVARIANCE. Scalar implicatures have traditionally been treated as a categorical phenomenon: either the implicature goes through or it does not. However, intuitively, implicatures are sometimes "felt" more strongly than other times (Russell 2012). Recent developments in probabilistic pragmatics have explicitly modeled scalar implicature as a matter of degree (Franke 2009, Russell 2012, Frank \& Goodman 2012, Degen, Franke \& Jäger 2013, Goodman \& Stuhlmüller 2013). In these models, hearers are treated as having a certain degree of belief in the stronger alternative being true or false upon observing an utterance containing a weak scalar item. This is akin to assigning the stronger alternative a particular probability of being true - the lower the probability, the stronger the implicature. ${ }^{5}$ Underlying these models is the assumption that hearers have internalized a model of the speaker, that is, of the utterances a speaker is likely to produce, given that the speaker intends to communicate a particular meaning. Bayesian inference allows hearers to then reverse-engineer a distribution over likely intended meanings, resulting in a probability (or degree of belief in) the stronger alternative being true or false. This stands in contrast to the traditional view, where the outcome of the reasoning process is a belief in the stronger alternative with minimal probability o or maximal probability 1 - in other words, the implicature goes through or it doesn't.

The sub-assumption of STRENGTH INVARIANCE captures not only that scalar implicatures are assumed to go through with probability o or 1, but also that they will always go through, with the exception of rare cases in which they are contextually canceled. This captures the consensus view both that scalar implicatures are a categorical phenomenon, and that they are rarely canceled, a view made explicit by various authors. For instance, Huang \& Snedeker (2009) hypothesize that "the lower-bounded [i.e., pragmatically unenriched] interpretation may be vanishingly rare in real-world communication". Horn (1984) remarks that "as a generalized implicatum, the aforementioned [scalar] inference goes through in unmarked contexts, but it may be canceled". Breheny, Katsos \& Williams (2006) note that scalar implicatures "show a degree of regularity and have the intuitive feel of components of conventional meaning".

5 In Bayesian cognitive science, it is commonplace to treat probabilities as degrees of belief in this way (Jaynes 1979). 
CONTEXT INDEPENDENCE. It is easy to see that if STRENGTH INVARIANCE holds, so does CONTEXT INDEPENDENCE; if the implicature arises irrespective of context, then context must play no role in whether or not a scalar implicature is derived. ${ }^{6}$ However, there are of course contexts in which the implicature is canceled, a fact often noted in the literature, but deemed to be a relatively rare occurrence (Levinson 200o). Under the strictest interpretation of the Homogeneity Assumption, then, CONTEXT INDEPENDENCE simply follows from STRENGTH INVARIANCE.

If there turns out to be more variability in implicature strength than expected, what is one to make of CONTEXT INDEPENDENCE? Given that there are cases in which the implicature is canceled, context must play some role in the process of computing scalar implicatures. Importantly, however, the role of context is not predicted to be systematic ${ }^{7}$ - cases of cancellation are in some way marked (Horn 1984) or idiosyncratic. Thus, under the strict interpretation of STRENGTH INVARIANCE, context should not play any role at all in scalar implicature computation. Under a looser interpretation that allows for some variability, context is allowed to play a role in idiosyncratic implicature cancellation, but implicature strength should nevertheless not be systematically predictable from features of the context.

Empirical predictions. The strictest form of STRENGTH INVARIANCE predicts that all utterances with some should be interpreted as giving rise to a some-not-all implicature. However, recent work (Frank \& Goodman 2012, Degen, Franke \& Jäger 2013, Goodman \& Stuhlmüller 2013) has provided evidence that scalar implicature is a probabilistic phenomenon; if we suppose that the process of interpreting an utterance with some can result in greater or lesser degrees of belief that the stronger alternative is false, then this provides a better fit to participants' judgments than it would if we instead assumed that an utterance either does or doesn't give rise to an implicature. Therefore, participants' task in the study reported below did not consist in simply giving categorical judgments. Instead, they were instructed to provide continuous implicature strength judgments. With this implicature measure, the strict version of STRENGTH INVARIANCE predicts all implicature judgments to be

6 Note that this entailment is asymmetric: if STRENGTH INVARIANCE holds, so does CONTEXT INDEPENDENCE. But if CONTEXT INDEPENDENCE holds, it is nevertheless possible for implicature strength to vary, e.g. because of noise processes in interpretation.

7 But see, e.g., Matsumoto 1995 for an attempt to capture systematically the conditions under which scalar implicatures are canceled. 
maximal. A looser version, taking into account that participants' judgments may be noisy and in some cases the implicature is canceled, predicts that implicature strength judgments should be generally high, but that in some exceptional cases, strength judgments may be low. That is, STRENGTH INVARIANCE predicts either a unimodal distribution of ratings clustered at the strong implicature end of the scale, or a bimodal distribution of ratings that is heavily skewed towards the strong end. The empirical pattern incompatible with STRENGTH INVARIANCE is a lack of preference for strong implicature judgments.

CONTEXT INDEPENDENCE is compatible with the patterns predicted by STRENGTH INVARIANCE. However, CONTEXT INDEPENDENCE is also compatible with substantial variability in participants' implicature strength judgments. Importantly, CONTEXT INDEPENDENCE predicts either that there should be no variability in strength, or if there is variability in strength, that this variability should not be systematically predictable from features of context.

If either or both of the sub-assumptions of the Homogeneity Assumption are not borne out by the data, this would have serious consequences for the status of implicatures from some to not all as GCIs. If there is a large amount of variability in implicature strength across contexts, some could not be said to "normally (in the ABSENCE of special circumstances) carry such-and-such an implicature or type of implicature", Grice's (1975) characterization of GCIs. If, moreover, variability in implicature strength is found to be systematically dependent on and predictable from context, some-not-all implicatures would start to smack suspiciously of PCIs. I return to the consequences this has for the status of the GCI-PCI distinction more generally in the general discussion in Section 3.

\subsection{An alternative view: Probabilistic pragmatics}

If the predictions of the Homogeneity Assumption are indeed not borne out, what would be an alternative framework within which to treat scalar implicatures and scalar implicature processing? Here I present a sketch of such a framework, which I will loosely refer to as probabilistic pragmatics. As with the traditional view of scalar implicatures as GCIs, I will present the probabilistic pragmatics framework in terms of the assumptions it makes. 


\section{(5) Probabilistic pragmatics}

a. Scalar implicatures are probabilistic.

b. Scalar implicatures are context-dependent.

c. Hearers can efficiently integrate multiple probabilistic contextual cues to the speaker's intended meaning.

In this framework, the problem of computing scalar implicatures is viewed from the hearer's perspective. Assumption (5a) reflects the view that scalar implicatures do not categorically either arise or fail to arise. Instead, as discussed above, scalar implicature strength reflects the hearer's resulting degree of belief in the stronger alternative being false (Frank \& Goodman 2012, Russell 2012, Degen \& Tanenhaus 2014). Multiple factors contribute to this ultimate belief: at least (a) the hearer's prior beliefs in the truth of the stronger alternative (world knowledge) and (b) the contextual evidence that the speaker intends to convey the negation of the stronger alternative. It is through the latter that assumption (5b) comes into play. Examples of contextual cues to the speaker's intention that will be investigated in Section 2.3 are the use of partitive of and the discourse accessibility of the NP referent embedded under some. The following case is an example of a partitive, highly discourse-accessible (as indicated by pronominalization) some-NP, which received high implicature strength ratings from experimental participants.

\section{(6) I sold some of them.}

Under this probabilistic view of scalar implicatures, implicature strength can vary, but this variation should be predictable from features of context. Hearers are assumed to have rich, probabilistic knowledge of the contexts in which speakers intend to communicate the negation of the stronger alternative. By making use of the available contextual information, the speaker's intention is reverse-engineered (or inferred) upon observing an utterance. This is assumption (5c) and another way in which the probabilistic pragmatics framework deviates from the traditional view that assumes that integration of contextual information is a difficult, cognitively costly process. Assumption (5c) is backed up by numerous findings from the psycholinguistic literature (Tanenhaus et al. 1995, Sedivy et al. 1999, Chambers, Tanenhaus \& Magnuson 2004, Heller, Grodner \& Tanenhaus 2008, Grodner \& Sedivy 2011).

Note that a consequence of this view of scalar implicatures is that the GCI-PCI distinction becomes obsolete. Each scale will likely be associated with different contextual features that hearers are sensitive to; and some 
may be more context-dependent than others. In consequence, conversational implicatures exhibit different degrees of context-dependence, instead of either being context-dependent or not. Under this view, the challenge lies in quantifying the cues that hearers are sensitive to in generating implicatures of varying strength and building explicit computational models of this process. This particular endeavor lies outside the scope of this paper, but see Russell 2012, Goodman \& Stuhlmüller 2013 and Bergen \& Goodman 2014 among others for examples of how contextual cues beyond utterance informativeness can be integrated into probabilistic models of scalar implicature.

\subsection{Interim summary}

In this section I have demonstrated how the Homogeneity Assumption follows from the GCI-PCI distinction, and worked out the two crucial empirical predictions it makes: (a) there should be little to no variation in implicature strength in implicatures from some to not all (but if there is, there should be a preference for strong over weak implicatures); and (b) to the extent that there is variability in implicature strength, it should not be predictable from or captured by features of context. I have also sketched an alternative view of scalar implicatures as a probabilistic, context-dependent computation problem for hearers. Section 2 reports the study conducted to test the Homogeneity Assumption for scalar implicatures from some to not all.

\section{Testing the Homogeneity Assumption}

In the following I report a corpus- and web-based study that constitutes a first attempt at testing the Homogeneity Assumption. The study tests two hypotheses. The first test consists in determining whether there is variation in participants' interpretation of utterances containing some. This constitutes a test of the STRENGTH INVARIANCE sub-assumption, which predicts little to no variation in implicature strength. The second test consists in determining whether there is systematicity to this variation; that is, whether certain features of the linguistic context reliably predict implicature strength. The CONTEXT INDEPENDENCE sub-assumption predicts that implicature strength should not be predictable from features of context. The contextual features, or cues to interpretation, as I will sometimes refer to them from the hearer's perspective, are: 
i. syntactic partitivity of the some-NP;

ii. determiner strength; and

iii. discourse accessibility of the some-NP, which includes

a. linguistic mention of the embedded NP referent,

b. topicality of the some-NP, and

c. modification of the head of the some-NP.

The study was conducted in three steps. First, a database of utterances containing some-NPs was generated by extracting all instances of utterances containing the word some from the Switchboard corpus. Second, implicature strength ratings were obtained for each case in the dataset via a web-based study. Finally, the obtained ratings were used to investigate the properties of interest: variation and systematicity in implicature strength.

\subsection{The database}

I used TGrep2 (Rohde 2005) to extract all 1748 occurrences of some-NPs that were not part of a disfluency from the Penn Treebank (release 3, Marcus et al. 1999) subset of the Switchboard corpus of telephone dialogues (Godfrey, Holliman \& McDaniel 1992). The corpus contains approximately 800 thousand spoken words in over 100 thousand utterances from about 650 telephone dialogues on various topics between two participants who did not know each other. The TGrep2 Database Tools (Jaeger 2006, Degen \& Jaeger 2011) were used to organize the some-utterances into a database.

Because only those cases that do not syntactically prohibit a scalar implicature were interesting for the purpose of the study, 359 cases (20.5\%) of some-NPs headed by singular count nouns were excluded. ${ }^{8}$

In a some-NP, singular count nouns are compatible with two different meanings. The more common meaning is the specific indefinite reading, which cannot give rise to a scalar inference (see examples in (7)). Singular count nouns in some-NPs can, however, also receive a coerced mass interpretation as shown in (8). Under this reading, the implicature, made explicit in (8b) is

8 In the grand scheme of things one would not want to exclude these cases of some, but rather include head noun number as a cue that hearers can use to restrict their interpretation of some - that is, a singular count noun can be seen as a strong, but nevertheless probabilistic, cue against the implicature. 
Distribution of scalar implicatures

possible, but these cases seem to be very infrequent (e.g., in a random sample of 50 singular count noun some-NPs, only three were cases of coercion, and they all occurred in the partitive, as in (9)).

(7) a. She stuck my name on some list.

b. *She stuck my name on some, but not all, list.

(8) a. John kicked some cat off the street.

b. John kicked some, but not all, cat off the street.

(9) Well, I had some of that problem.

A further 26 cases where the some-NP consisted only of some were also excluded:

(10) Some say that coffee is healthy.

This was done because for these cases it is not possible to investigate the effects of the discourse accessibility cues tested in Section 2.3, which assumes that some occurs with an embedded NP. However, it is worth noting that in these cases the implicature seems to generally go through.

After the exclusion, 1363 cases of utterances containing some-NPs remained. For these cases, implicature ratings were collected in a web-based study, which is described in the following section.

\subsection{Collecting implicature ratings: a test of STRENGTH INVARIANCE}

Gradient implicature strength ratings were collected using Amazon's Mechanical Turk service. ${ }^{9}$

\subsubsection{Methods}

Participants 243 participants were recruited on Amazon's Mechanical Turk and paid \$o.8o for each block of 20 items. Participants who completed at least three blocks received a one-time bonus of \$o.20.

9 The experiment can be viewed at https://www.hlp.rochester.edu/mturk/jdegen/7_qpsome/ output/qp.html?assignmentId=foo\&list=1. Different lists can be viewed by changing the list parameter to any number between 1 and 67. The data, and the R code for generating the figures and analyses, are available at https://github.com/thegricean/corpus_some. 
Procedure and materials On each trial, participants saw an utterance ${ }^{10}$ containing a some-NP (the target utterance) together with ten utterances from the immediately preceding discourse context (or until the beginning of the dialogue if there were fewer than ten utterances in the previous context). The target utterance was presented in red. Below the target utterance, an almost identical utterance (the comparison utterance) was presented which differed only in that the implicature was made explicit by inserting but not all after some. The comparison utterance was presented in green font. Two example pairs of (a) target and (b) comparison utterances are shown in (11) and (12).

(11) a. I like, I like to read some of the philosophy stuff.

b. I like, I like to read some, but not all, of the philosophy stuff.

(12) a. And I'll take some time and do that with her.

b. And I'll take some, but not all, time and do that with her.

Participants were then asked, "How similar is the statement with 'some, but not all' (green) to the statement with 'some' (red)?" They provided similarity judgments on a seven point Likert scale with endpoints labeled as "very different meaning" and "same meaning" and individual points labeled as $1,2, \ldots, 7$. The more clearly the implicature is part of the speaker's originally intended meaning, the less of a difference explicitly encoding the content of the implicature should make, and the higher the similarity judgments are expected to be. Conversely, if the content of the implicature was not part of the speaker's originally intended meaning, making it explicit should lead to a larger perceived shift in meaning and the two utterances should be rated as very dissimilar.

This paraphrase task is a novel measure of scalar implicature and as such deserves further consideration. I briefly discuss three task-related considerations.

First, the effect of locally inserting but not all should have different effects on the interpretation of some in upward-entailing versus non-upwardentailing contexts (Chierchia 2004). While this is an important point that deserves further investigation in future work, ${ }^{11}$ see Section 2.3.2 for a demon-

10 An utterance corresponds to a unit of speech that has been transcribed as a sentence in the Switchboard corpus. This includes sentence fragments.

11 To the best of my knowledge, there exists no large-scale empirical assessment of the rate at which various scalar items occur in upward-entailing versus non-upward-entailing contexts in naturally occurring language. 
stration that in the dataset reported here, monotonicity properties of the context likely affected only a negligibly small number of cases.

A further consideration is that inserting but not all may shift the salient Question Under Discussion (QUD) (Roberts 2012) in some cases but not others. That is, participants' judgments may in some cases reflect not implicature strength, but the difficulty of making reparatory inferences to accommodate the shifted QUD. While it is quite likely that judgments reflect QUD accommodation in some cases (e.g., in cases like the ones listed in (15)), this is not at odds with the notion that these judgments reflect implicature strength. In fact, relevance of the stronger utterance with all to a salient contextual QUD is a crucial ingredient in scalar implicature computation (Grice 1975, Matsumoto 1995, Zondervan 2010, Russell 2012). Under the probabilistic view of scalar implicatures, relevance of the stronger alternative to a contextual QUD is one of many factors involved in scalar implicature. Thus, reduced implicature strength due to failed QUD accommodation is perfectly compatible with the view taken in this paper. It is nevertheless an interesting question for future work whether QUD accommodation processes can be teased apart experimentally from "core" implicature computation.

A final consideration regards participants' potentially varying interpretation and resulting use of the provided Likert scale. In order to effect similar scale interpretations, participants were first familiarized with the task and scale range by completing two practice trials before completing the experimental trials. One of the practice trials was a clear case of a scalar implicature, shown in (13), while the other one, shown in (14), clearly could not give rise to the relevant implicature. Each practice utterance was presented in context (see Appendix A).

(13) a. I had some of the banana yogurt.

b. I had some, but not all, of the banana yogurt.

(14) a. There are probably some peanuts in the pantry.

b. There are probably some, but not all, peanuts in the pantry.

Participants were told that cases like (13) should receive a high rating and cases like (14) should receive a low rating, but were not instructed on which particular value to assign.

Items were divided into blocks of 20 items each. Each block was rated by ten participants. Eleven items appeared in two different blocks in order to ensure that each block consisted of 20 items. Because of this, most items received 10 ratings each and 11 items received 20 ratings each. 


\begin{tabular}{lllllllllllllllllllllll}
1 & 2 & 3 & 4 & 5 & 6 & 7 & 8 & 10 & 12 & 14 & 16 & 18 & 20 & 22 & 24 & 26 & 34 & 44 & 46 & 48 & 54 & 100 \\
\hline
\end{tabular}

$\begin{array}{lllllllllllllllllllllll}26 & 120 & 3 & 12 & 2 & 35 & 1 & 13 & 6 & 3 & 3 & 5 & 2 & 2 & 1 & 1 & 1 & 1 & 2 & 1 & 1 & 1 & 1\end{array}$

Table 1 Distribution of participants (bottom rows) over completed number of blocks (top rows).

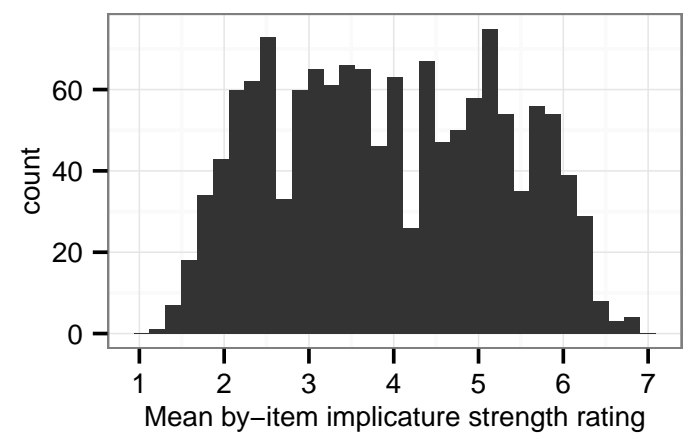

Figure 1 Distribution of mean per-item implicature strength ratings. ${ }^{12}$

\subsubsection{Results and discussion}

The distribution of participants over number of rated blocks of items is shown in Table 1. Mean number of completed blocks per participant was 5.72 (median: 2).

Mean overall similarity rating was 3.9 (median: 4.0). The distribution of raw ratings and (aggregated) mean by-item ratings is shown in Figure 1. Under the Homogeneity Assumption - in particular the STRENGTH INVARIANCE subassumption - there should be more high than low ratings. Indeed, ratings should be clustered at the upper end of the scale, reflecting overall strong support for the implicature. However, only $44.7 \%$ of ratings were higher than the midpoint of the scale, while $46.6 \%$ of ratings were lower than 4 . Looking only at the endpoints of the scale, only $14.7 \%$ of the data were highest ratings while $19 \%$ were lowest ratings. Thus, contrary to STRENGTH INVARIANCE, there is a substantial amount of variation in implicature strength across items.

12 The similarity ratings obtained in this study will generally be referred to as ratings of implicature strength, which is what they are intended to capture. For a discussion of other factors that could be affecting the ratings, see Section 2.2.1. 
Distribution of scalar implicatures

Examples from the lower, medium, and upper end of the scale are shown in (15-17). Numbers on the right indicate mean similarity rating.

(15) Low similarity rating (little support for implicature)

a. That would take some planning. ${ }^{13} \quad 1.4$

b. And this would give them a chance to have some positive selfesteem.

c. You sound like you've got some small ones in the background. 1.5

(16) Medium similarity rating (medium support for scalar implicature)

a. And some ways, it might be kind of scary. 4.0

b. I'd love to have, have some animals. 4.0

c. It would certainly help them to appreciate some of the things that we have here $\quad 4.0$

(17) High similarity rating (much support for implicature)

a. But I think that at some times it can be the right thing to do. 6.7

b. I sold some of them. $\quad 6.8$

c. I like some country music. $\quad 6.9$

This amount of variation in implicature strength is quite unexpected from the perspective of the previous literature, which overwhelmingly makes the Homogeneity Assumption. These results constitute a good example of how empirically studying a large group of linguistically untrained language users' pragmatic judgments about naturally occurring language can yield very different results from received wisdom based on individual researchers' intuitions about artificial examples.

By the same token, as this is the first study of its kind, ${ }^{14}$ it is important to address potential effects of various methodological choices on the outcome of the study. One potential concern is that when given a gradient scale on which to provide judgments, participants will use the entire scale even if they don't perceive great meaning differences between items. That is, it is possible that in fact, participants strongly got the upper-bound reading in

13 Throughout the paper, where examples are taken from the corpus, the some-NP is highlighted in boldface.

14 Though see Doran et al. 2012 and van Tiel et al. 2014 for attempts to quantify between-scale variation in scalar implicature strength and between-implicature-type variation in implicature strength, respectively. 
every case, but distributed their judgments over the scale in order to avoid "wasting" the scale. ${ }^{15}$ If control items that differed more strongly in meaning had been included in the items, perhaps participants' judgments would have actually been clustered at the upper end of the scale, as predicted by the Homogeneity Assumption.

This raises a more general issue for experimental pragmatics: the susceptibility of participants' pragmatic judgments to both (a) the other items included in the experimental stimuli and (b) the dependent measure used to collect judgments. I suspend discussion of the more general issue here ${ }^{16}$ and instead focus on how one could test whether participants distributed their judgments over the scale for the uninteresting and theoretically misleading reason that they wanted to use the entire scale, or for the interesting reason that they perceived the implicature with varying strength.

Suppose that participants simply wanted to use the entire scale. In this case, there are no systematic reasons for giving an item a high or a low rating; scale use should be random. If this is so, there should be no systematicity to the strength of participants' judgments. Each item should have received wildly different (random) ratings from different participants, resulting in byitem means clustered around the midpoint of the scale. ${ }^{17}$ This is not borne out in the data: some items received very low means, some items very high ones. Furthermore, it is encouraging for the validity of the paraphrase measure that for many cases (e.g., the examples listed in (15-17)), the empirically obtained results are in line with intuition. However, future work should investigate the consequences of using different dependent measures to collect implicature judgments for these items. ${ }^{18}$

15 Another possibility is that in some cases, the comparison utterance with explicit but not all was either contextually infelicitous or even ungrammatical because the alternative with all was not contextually available, e.g. in (15a). In this case, low ratings might reflect ungrammaticality/infelicity of the comparison utterance rather than a large (but not trivially so) difference in meaning. Future work should estimate the extent to which the stronger alternative is available for each item, but an in-depth investigation of this issue is beyond the scope of the current paper.

16 But see, e.g., Degen \& Goodman 2014 for an investigation of different dependent measures' varying sensitivity to context effects in the domain of scalar implicature.

17 That this is so can easily be shown by a simple simulation treating each item mean as the result of 10 random samples drawn from a 7-point Likert scale. For 1363 items, this yields a Gaussian distribution with a global mean of 4 and standard deviation of 0.6 , which is very different from the observed distribution. See Appendix B for details.

18 See Geurts \& Pouscoulous 2009 and Degen \& Goodman 2014 for discussion of dependent measure choice in experimental pragmatics. 
Distribution of scalar implicatures

A second prediction that emerges if participants did not use the scale systematically is that the variation in item means should not be predictable from contextual features. This prediction is in alignment with the prediction made by the Homogeneity Assumption's sub-assumption of CONTEXT INDEPENDENCE, which will be tested in Section 2.3.

This section reported a test of the STRENGTH INVARIANCE sub-assumption of the Homogeneity Assumption. The results revealed a much greater degree of variation in implicature strength for implicatures from some to not all than expected under STRENGTH INVARIANCE, suggesting the assumption is not warranted. The next section tests the second sub-assumption, that of CONTEXT INDEPENDENCE.

\subsection{Analyzing the role of contextual cues in implicature strength: a test of CONTEXT INDEPENDENCE}

I next turn to investigations of the individual and joint effects of different contextual cues on scalar implicature strength, none of which are predicted to have an effect if implicatures from some to not all constitute a homogeneous class of context-independent implicatures. The investigated cues are (a) the partitive form, (b) determiner strength, and (c) discourse accessibility as quantified by linguistic mention, topicality, and modification. I discuss each of these in turn.

\subsubsection{Cue 1: the partitive form}

Consider the difference between (18) and (19).

(18) Alex ate some of the cashews.

(19) Alex ate some cashews.

Intuitively, there is a clear difference in how strongly each of these utterances gives rise to the implicature that Alex did not eat all the cashews. In the example with the overt partitive form of, intuition strongly suggests that Alex did not eat all the cashews, while in the example without the partitive this intuition is much weaker. Before addressing whether these intuitions are substantiated in the empirical data, it is worth discussing reasons why the partitive has this effect.

First, it is well-known that there is an additional constraint on using the partitive structure that is not in play for non-partitive quantifiers. Jackendoff 
(1977) originally formulated the constraint as one of definiteness of the NP embedded under some (of):

\section{(20) Partitive Constraint I}

The complement NP in a partitive must be definite.

Subsequently, this formulation of the constraint was shown to be too strong: there are well-documented cases of indefinite but specific partitives, as in one of many people who saw the accident or half of a cookie (Ladusaw 1982).

Reed (1991) re-formulated the constraint as one of discourse accessibility. She proposed that the embedded NP must refer to a discourse accessible group; rather than evoking a discourse group, the embedded NP must refer back to an already mentioned (or inferable) discourse group. The function of the partitive structure is to evoke a subgroup of that discourse group. Under a discourse accessibility account like Reed's, the strong preference for the embedded NP to be syntactically definite is explained by the embedded NP's discourse function: "the need to access a discourse group creates a preference for, but not a restriction to, definite NPs in the embedded position” (Reed 1991, p. 216).

Whence, then, the intuition that partitive some more strongly gives rise to the implicature that Alex did not eat all of the cashews than non-partitive some? Consider what drawing the implicature requires. In order to infer that the speaker intended to convey that $X$ is the case of some, but not all, $Y$, there must be some group $Y$, mutually known by both interlocutors, that can be partitioned. Such groups are precisely Reed's “discourse accessible groups". That is, the partitive's intuitively high propensity to give rise to scalar implicatures is a consequence of the discourse accessibility constraint on NPs embedded under partitives. It is only with discourse accessible NP referents that scalar implicatures should be able to arise.

Note that this does not prevent utterances with non-partitive some from giving rise to scalar implicatures. That is, using the partitive is not necessary to get scalar implicatures from utterances with some. As long as the embedded NP is discourse accessible, the scalar inference is possible, whether or not the some-NP is overtly partitive. For example, it seems that if (19) was uttered in a context with a contextually given set of cashews, the speaker should more strongly be taken to mean that Alex did not eat all the cashews than if such a set was not given. 
Distribution of scalar implicatures

The a priori difference between partitive and non-partitive some in how strongly they are associated with a scalar implicature can be summarized as follows: Scalar implicatures can only arise with discourse accessible embedded NP referents. The partitive structure can only be used with discourse accessible embedded NP referents, while non-partitive some can be used with both accessible and inaccessible referents. Thus, the a priori probability of a scalar implicature is higher for partitive some (which always occurs with accessible embedded NP referents) than for non-partitive some (which only sometimes occurs with accessible embedded NP referents).

However, the occurrence of the partitive itself is not sufficient for a scalar implicature to arise, either. Recent evidence from experiments on the processing of scalar implicatures provides some support for this claim. Degen \& Tanenhaus (2014) found higher implicature rates for statements with partitive some than for those with non-partitive some in a truth-value judgment task. However, implicature rates were not at 100\% for either construction, suggesting that the partitive does not categorically force the proper part reading, as has often been noted in the literature (e.g., Horn 1997).

Thus, the presence of the partitive should be a strong but nevertheless probabilistic cue that increases implicature strength, but does not fully determine it, compared to cases where the partitive form is absent.

Data analysis Here and in the following, I report the results of linear mixedeffects regression models (Baayen, Davidson \& Bates 2008) to test the effect of different cues on implicature ratings while simultaneously accounting for conditional dependencies between data points from the same rater. ${ }^{19}$ These dependencies are captured in so-called random effects, which offer a convenient way to account for violations of the assumption of independence of each data point (for an introduction directed at language researchers, see Jaeger 2008). This kind of independence cannot be assumed for datasets in which different participants contribute multiple data points; in our case, different participants may have systematically different perceptions of how large the shift in meaning is when the implicature is made explicit. Thus a forgiving participant may have given systematically higher similarity ratings than another, less forgiving, participant. More generally, there may be differences in how different participants use the rating scale. Random effects

19 Performing ordinal regression, which accounts for the fact that the obtained data were discrete judgments from a Likert scale, yields the same qualitative results (in terms of significance of effects) as the linear mixed effects model reported here. 
can account for this individual participant variability and thus crystalize the effects of the cues under investigation.

All statistical analyses used mixed-effects linear regression models predicting implicature strength rating from fixed effects of interest (the cues under investigation) and the following random effects structure: random by-participant intercepts, random by-participant slopes for all fixed effects, and random by-item intercepts. ${ }^{20}$ All fixed effect predictors were centered before entering the analysis. Results were obtained using the 1 me4 (Bates et al. 2014) and 1 merTest (Kuznetsova, Brockhoff \& Christensen 2014) packages in $\mathrm{R}$ (Team 2014). The partitive and the determiner strength predictor were allowed to interact, as were the three discourse accessibility predictors. I report the main effect of each cue individually. The interaction between partitive and determiner strength is discussed in Section 2.3.2. The interaction between the different discourse accessibility predictors is discussed in Section 2.3.3. The full model is summarized in Appendix D.

Results The dataset of 1363 cases contained 368 (27\%) partitives, of which in turn $26.8 \%$ were headed by pronouns or demonstratives as in (21) and (22).

(21) Uh, some of that unfortunately is legal.

(22) And for some of them it was just kind of, I don't know, not so much a holiday.

As can be seen in Figure 2, the overtly partitive cases received higher implicature ratings than the non-partitive cases $(\beta=0.91, S E=0.09, t=9.6$, $p<.0001$ ). Compared to the global mean rating of 3.9, the partitive mean was higher at 5, while the non-partitive mean was lower at 3.5. Similarly, the median rating for partitive cases was 5 , while the non-partitive median was 3 .

20 Barr et al. 2013 recommend using the maximal random effects structure whenever possible. In this case, random slopes were only included for participants because each item occurred with only one value of each fixed effect - this is a feature inherent to naturalistic corpus data, where the experimenter is "given" items by nature rather than creating and tightly controlling items by presenting them to participants in different conditions. In consequence, variability in by-item random slopes for the fixed effects in this study cannot be estimated reliably. In keeping with Barr et al. 2013, the model thus included the maximal random effects structure that was possible.

21 Here and throughout the rest of the paper, error bars indicate bootstrapped 95\% confidence intervals and numbers in bars indicate number of contributing cases. 

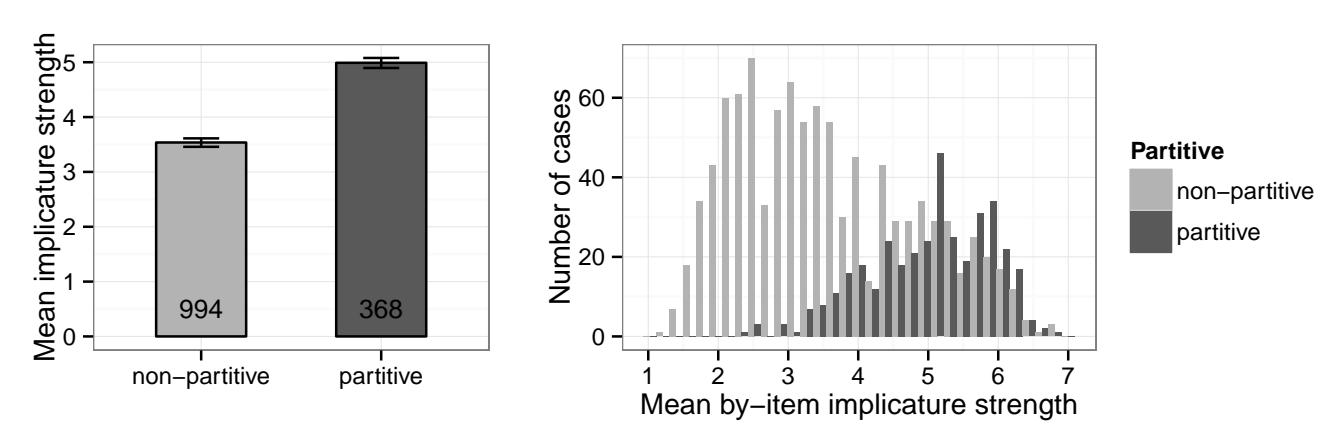

Figure 2 Mean implicature strength ratings (left) and distribution of mean by-item ratings (right) for non-partitive and partitive some-NPs. ${ }^{21}$

While $44.7 \%$ of cases globally received ratings above the midpoint of the scale, conditioning on overt partitivity (i.e., excluding non-partitive cases) increases that number to $67.8 \%$. This suggests two things: (a) the Homogeneity Assumption seems to be more warranted when the some-NP is overtly partitive; and (b) the partitive is nevertheless not sufficient for unambiguously generating a scalar implicature - only $25 \%$ of ratings were $7 \mathrm{~s}$, and $23 \%$ of ratings were still below the midpoint of the scale. Examples of partitive cases that received low similarity ratings are shown in (23-25) alongside their mean similarity ratings.

(23) I wish my mother had had some of those opportunities, because, I think she would have really, she rea-, would have succeeded in a lot of ways, that men, that women were not able to succeed in her generation.

(24) But when you get into some of these health clubs where you just stand around and wait...

(25) I just go to be entertained and am not really interested in some of the, like, the Terminator or some of the Schwarzenegger stuff.

In all three cases, the implicature is not licensed (or only very weakly so) despite the presence of the partitive. 


\subsubsection{Cue 2: determiner strength}

The word some is ambiguous between a weak, indefinite, or non-presuppositional reading, often written as $s m$ because it tends to be unstressed, and a strong, quantificational, or presuppositional reading (Milsark 1974, 1977, Barwise \& Cooper 1981, Ladusaw 1994, Israel 1999). Consider the example in (26).

(26) Some prospectors got the plague.

The sentence in (26) can mean either that there is an indefinite number of prospectors who got the plague (weak, sometimes also called cardinal interpretation) or that some prospectors got the plague but others presumably did not (strong, sometimes also called partitive or proportional interpretation). In general, determiners can either be unambiguously weak (e.g., a/an and no) or strong (e.g., all and most), or ambiguous between the two readings (e.g., some).

The distinction between weak and strong determiners is central to the distribution of scalar implicatures from some to not all because it has been noted that the use of strong, but not weak, determiners gives rise to scalar implicatures (Ladusaw 1994). Indeed, the partitive form (which, as noted in the previous section, is associated with higher implicature rates than non-partitives) tends to only occur with strong determiners (e.g., Horn 1997, Ladusaw 1994).

However, the weak/strong distinction has been notoriously difficult to pin down (e.g., Horn 1997). The goal here is not to give an exhaustive review of the rich literature on weak and strong determiners, but rather to identify an operationalization of the weak/strong distinction that will facilitate a quantitative test of whether strong some is more likely to give rise to scalar inferences than weak some. To foreshadow, the presuppositionality difference between weak and strong some-NPs (e.g., Lumsden 1988) will be employed to arrive at empirical ratings of the strength of each use of some in the database. I begin by elaborating on some of the properties that have been observed to correlate with the distinction.

Table 2 summarizes the diagnostic tests relevant for our purposes, provided in a review by Horn 1997. The property that we crucially depend on in collecting strength ratings from participants is one suggested by de Jong \& Verkuyl (1985) and Lumsden (1988) among others. They propose that strong determiners introduce the presupposition that their restriction is not empty 
Distribution of scalar implicatures

\begin{tabular}{lll}
\hline \multicolumn{2}{c}{ Strong some } & \multicolumn{1}{c}{ Weak some } \\
\cline { 2 - 3 } (a) & presuppositional & non-presuppositional \\
(b) & partitive or proportional & cardinal \\
(c) & scalar implicatures likely & scalar implicatures unlikely
\end{tabular}

Table 2 Diagnostics for identifying strong versus weak uses of some (based on Horn 1997).

and their domain of quantification is part of the domain of discourse. That is, under the strong interpretation of (26), there needs to be some set of prospectors in the domain of discourse of whom it is being predicated that they got the plague. Under the weak reading, the domain of discourse need not contain a set of prospectors - the set is introduced (the discourse group evoked, in Reed's (1991) terms) by the some-NP.

The weak/strong distinction correlates with other properties which are not directly relevant to our purpose of finding an empirical operationalization of the weak/strong distinction - for instance, the propensity to occur in existential there constructions (Milsark 1974, McNally \& Geenhoven 1998) and the ability to occur with individual-level predicates (Carlson 1977, Milsark 1977). Importantly, the literature provides counterexamples to each of these diagnostics (see e.g., Horn 1997, McNally \& Geenhoven 1998). Rather than being strict constraints or part of the definition of strong determiners, it seems that these properties are approximate diagnostics and I will treat them as such.

In particular, to arrive at an estimate of the strength of some for each of the cases in the database, the presuppositionality difference was exploited in a web-based study collecting participants' judgments about the use of some. ${ }^{22}$ To quantify determiner strength, participants rated the similarity of each original utterance from the dataset to the same utterance without some (of) on a seven-point Likert scale. The reasoning behind this choice was built on the presuppositional nature of strong NPs: the weak use of some does not have a non-empty restriction presupposition associated with it, while the strong one does. Thus, in removing some (of), the change in meaning should be greater for strong than for weak some-NPs. Consider examples (27) and (28).

22 Details of this study can be found in Degen 2013. 
(27) Weak use

a. But my son needed sm money.

b. But my son needed money.

(28) Strong use, partitive

a. And some of the people in our church use birth control.

b. And the people in our church use birth control.

(29) Strong use, non-partitive

a. Some history books are pretty scary.

b. History books are pretty scary.

Mutual entailment holds between the (a) and (b) sentences in (27) but not in (28) and (29); that is, all else being equal, the difference in meaning between the (a) and (b) forms in (28) and (29) is greater than in (27). Thus, the higher the similarity rating given for a particular case, the weaker the use of some in this case. Conversely, the lower the rating, the stronger the use.

Results The distribution of mean by-item strength ratings is shown in the left panel of Figure $3 .{ }^{23}$ Before turning the effect of determiner strength on implicature strength, it is important to investigate the quality of the obtained determiner strength ratings by testing whether they correlate with the diagnostics proposed in the literature. Here we report only the correlation of determiner strength with partitivity. The right panel of Figure 3 demonstrates that partitive cases received on average much lower similarity ratings (i.e., higher strength ratings) than non-partitive cases. However, no bimodal distribution indicating two categorically distinct uses - weak versus strong - was observed, supporting the decision to treat determiner strength as a continuum.

For further evidence that the determiner strength ratings obtained here correlate with other diagnostics proposed in the literature (e.g., the aversion of strong determiners to occurring in existential there constructions or the strong tendency for weak determiners not to occur with individual-level

23 Note that in this study, strength ratings were not collected for the 99 cases where the head of the embedded NP was a deictic expression like a pronoun or a demonstrative. Thus, strength ratings were not independently available for these cases, but were instead simulated in a principled way. See Appendix C for details of the procedure. 
Distribution of scalar implicatures

predicates), see Degen 2013. We can now turn to the effect of determiner strength on implicature strength.

Implicature ratings were lower with decreasing determiner strength $(\beta=$ $-0.5, S E=0.05, t=-9.5, p<.0001)$. This is shown in Figure 4 . The stronger the use of some, the stronger the support for a scalar inference. Conversely, the weaker the use, the weaker the implicature. ${ }^{24}$ This is compatible with the general observation in the literature that strong uses of some can give rise to the implicature, but it is important to note that this is not a perfect correlation (Pearson's $r=-.51$ ). ${ }^{25}$ That is, some uses of the determiner were judged as strong but did not strongly support the implicature, whereas others were judged to be weak but nevertheless received high implicature strength ratings. Examples of each of these cases are given in (30) and (33).

\section{(30) Strong determiner, low implicature rating}

a. I'd like to go to Sundance and Park City and some of those.

$2.6 / 3.6$

b. What are some of the things they don't recycle.

c. Maybe this would be a way to get that feeling back, if we've lost some of that.

(First number: mean determiner strength rating; second number: mean implicature rating.)

Cases of strong some that nevertheless give rise to scalar implicatures only weakly, if at all, are not in principle surprising: standard lower-bound interpretations, where the implicature does not arise because the stronger alternative is not contextually relevant, should give rise to just this pattern.

24 There was also an interaction of determiner strength and partitivity of the some-NP ( $\beta=0.39$, $S E=0.1, t=4.1, p<.0001)$. One potential reason for this interaction is that determiner strength has an effect on implicature strength for non-partitive (potentially weak or strong) cases, but not for partitive (i.e., by definition, strong) cases. However, inspecting the simple effects model reveals that there is an effect of determiner strength on both partitive and nonpartitive cases; the significance of the interaction term arises from the effect of determiner strength being weaker for partitive than non-partitive cases. This provides further evidence that even within partitives, there are stronger and weaker cases of some.

25 Note that the correlation is negative because higher ratings in the determiner strength rating task corresponded to weaker uses of the determiner and conversely, lower ratings indicated stronger determiner use. Thus, high implicature ratings should be correlated with low determiner strength ratings, resulting in a negative correlation - which is what we observe. 

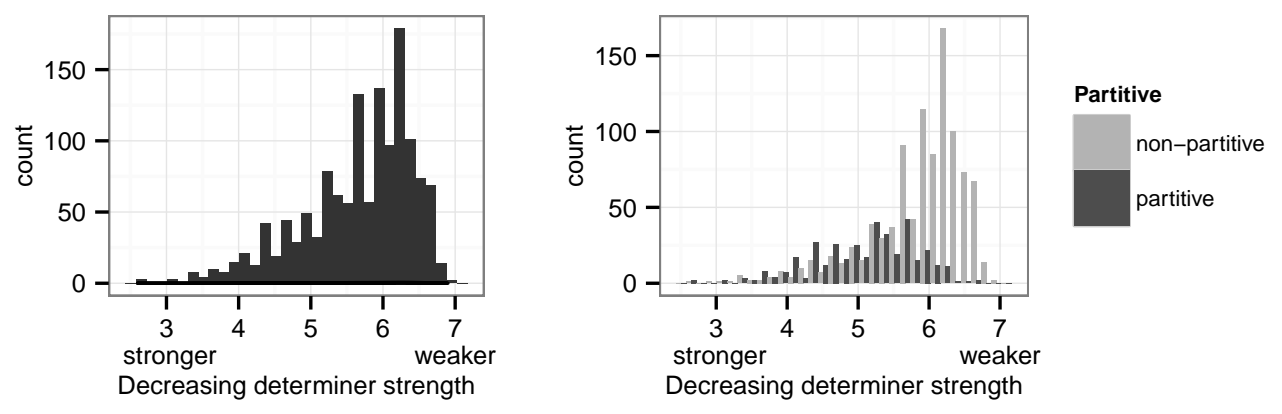

Figure 3 Distribution of mean by-item determiner strength ratings overall (left) and conditioned on whether or not the some-NP was overtly partitive (right). Higher ratings indicate weaker determiner uses.

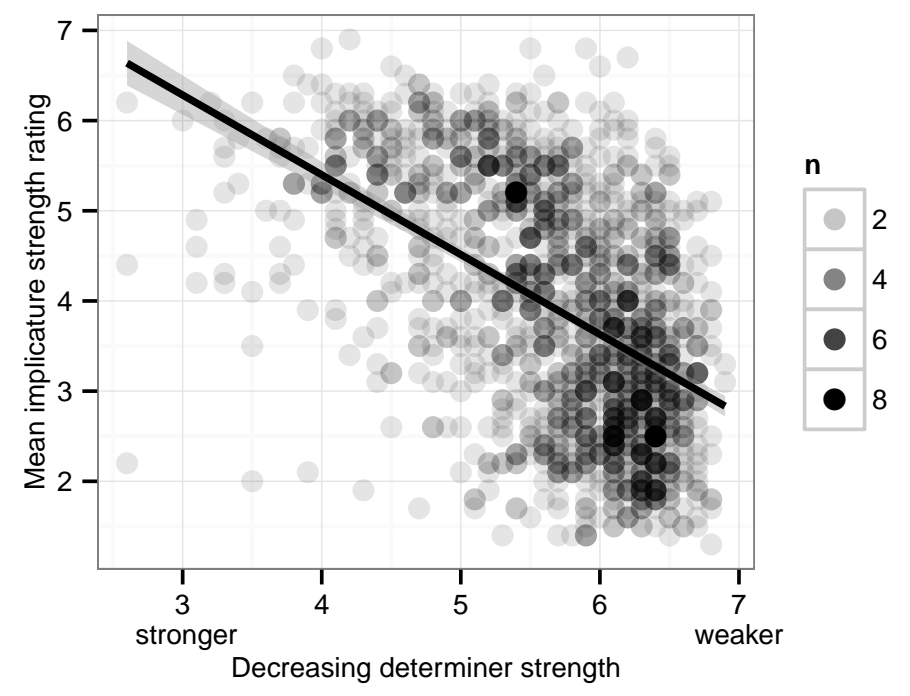

Figure 4 Mean by-item implicature rating as a function of decreasing determiner strength. Opacity of each point indicates the contributing number of data points (i.e. darker dots indicate more contributing cases).

The example in (30a) seems to be of this type. In contrast, the weak implicature support in (30b) and (30c) seems to have a different source: in (3ob), the some-NP is embedded in a wh-question, while in (30c) it is in the antecedent 
Distribution of scalar implicatures

of a conditional. Both of these are instances of non-upward-entailing environments, which have been known to cancel and even flip implicatures (Atlas \& Levinson 1981, Horn 1989, Levinson 2000, Chierchia 2004, Chierchia, Fox \& Spector 2008).

Context monotonicity annotation of a random sample of 50 cases yielded only two cases where the some-NP occurred in non-upward-entailing contexts; both of these were polar interrogatives. If this is a good estimate of the rate at which some occurs in these contexts, roughly $4 \%$ of some-NPs occur in nonupward-entailing environments. In these cases, implicature ratings should be low. The following two are the polar interrogative cases with their mean implicature strength rating.

(31) Or do some of them play the same song? 4.7

(32) But is it a legal, uh, solution for some companies? 5.4

Both of these mean ratings are higher than the global mean in the dataset, suggesting that at least in polar interrogatives, the implicature is not categorically ruled out. However, a complete test of the effect of non-upward-entailing (and especially downward-entailing) contexts on ratings in this dataset remains to be conducted.

I turn next to examples of cases where determiner use was judged as weak but implicature ratings were nevertheless high.

(33) Weak determiner, high implicature rating

a. It's hurting, you know, it's hurting Germany, for example, too, and some other parts of Europe where they, where they have high industry.

$6.4 / 5.7$

b. And, after I, I graduated, I read some of the old classics that I just bluffed my way through and have found that I enjoy them quite a bit, too.

$6.2 / 6.0$

c. But I think that at some times it can be the right thing to do.

$6.2 / 6.7$

d. And then on the other hand, I've seen some people go into the nursing home and just so happy you know.

$5.8 / 5.7$

(First number: mean determiner strength rating; second number: mean implicature rating)

There seem to be two different things going on here. In (33a) and (33b), use of the determiner is weak in that it is introducing two new discourse groups: 
other parts of Europe and old classics. However, the modifying post-nominal material introduces a contrast with a (presumably non-empty) complement set: parts of Europe where they don't have high industry and the old classics that I did not bluff my way through. In these cases, then, the upper-bound interpretation may not arise as a standard implicature, but as a consequence of the non-empty complement set presupposition introduced by the postnominal modification.

Similarly, in (33c) and (33d) the upper-bound interpretation does not seem to be due to the standard Quantity reasoning, but instead is due to the fact that the prior probability of the state of the world signaled by the upperbound interpretation is high: world knowledge tells us that it is more likely that it is not at all times the right thing to do rather than that it is (whatever it may refer to in this case). And it is more likely that not all people go into the nursing home and are happy rather than that they all are.

Thus, while implicature support is strongly correlated with determiner strength, factors like monotonicity properties of the context that the some-NP is embedded in, discourse expectations, and world knowledge affect scalar implicatures.

\subsubsection{Cue 3: Discourse accessibility}

As discussed above, Reed (1991) proposed a discourse accessibility constraint on the partitive: the partitive can only be used with embedded NPs referring to discourse accessible referents. Relatedly, strong uses of some have been argued to be covertly partitive and to have a discourse accessibility presupposition on the embedded NP. In this section I investigate the effect of discourse accessibility on scalar implicatures above and beyond overt partitivity and determiner strength.

Several factors contribute to discourse accessibility: here I investigate (a) linguistic mention of the embedded NP referent, (b) topicality of the some-NP, and (c) modification of the embedded NP.

Several researchers have noted that scalar implicatures seem to be affected by information structure. For example, Breheny, Katsos \& Williams (2006) found that more scalar implicatures are generated in Greek when the some-NP is in subject position than when it is in object position. Their explanation is that scalar implicatures should only arise for sentences that address a contextual QUD that is about the constituent containing the scalar item. Because of the strong tendency of Greek (and weaker tendency of En- 
glish) for subjects to contain old information, i.e. information that a QUD is about, scalar implicatures should be more likely to arise for some-NPs in subject position than in positions that are lower on the obliqueness hierarchy (e.g. objects, adjuncts, etc.). ${ }^{26}$

Taken together, this predicts effects of both linguistic mention and subjecthood on scalar implicatures: implicature ratings should be higher with previously mentioned some-NPs and with subject some-NPs. Additionally, adding pre- or post-nominal modification to an NP that refers to a new (previously unmentioned) entity or group makes this group accessible (Reed 1991). Consider the following example:

(34) When we arrived at the hotel we didn't know where to go so we asked the guy at the front desk.

The restrictive modifier at the front desk makes the novel mention of the guy discourse-accessible by providing uniquely identifying information (Webber 1983, Reed 1991).

The combination of these three different markers of discourse accessibility - mention, topicality, and modification - could plausibly affect scalar implicature strength in various ways. First, it is possible that each of the markers has an independent, additive effect. ${ }^{27}$ For example, an utterance with a modified subject some-NP may more strongly give rise to the implicature than one with an unmodified subject some-NP. This would constitute evidence for a gradient notion of discourse accessibility: the more discourse-accessible a particular some-NP, the stronger the implicature. Another possibility is that discourse accessibility affects implicature strength in a categorical way, such that as long as the discourse accessibility of the some-NP is guaranteed by at least one of the markers (subjecthood, previous mention, or modification), the presence of another marker has no further strengthening effect on the implicature.

To reflect the potential for complex interactions between discourse accessibility markers, predictors for linguistic mention, subjecthood, and modification were allowed to interact in the regression model. I first discuss the

26 Note, however, that other accounts make the opposite prediction. For example, van Kuppevelt (1996) proposes that scalar implicatures can only arise if the scalar item occurs in the comment part of the sentence, that which answers the contextual QUD. However, the default comment position in English is the object position.

27 This is assuming that each of the markers has some effect. It is of course also possible that none of them or only a subset affects implicature strength. 

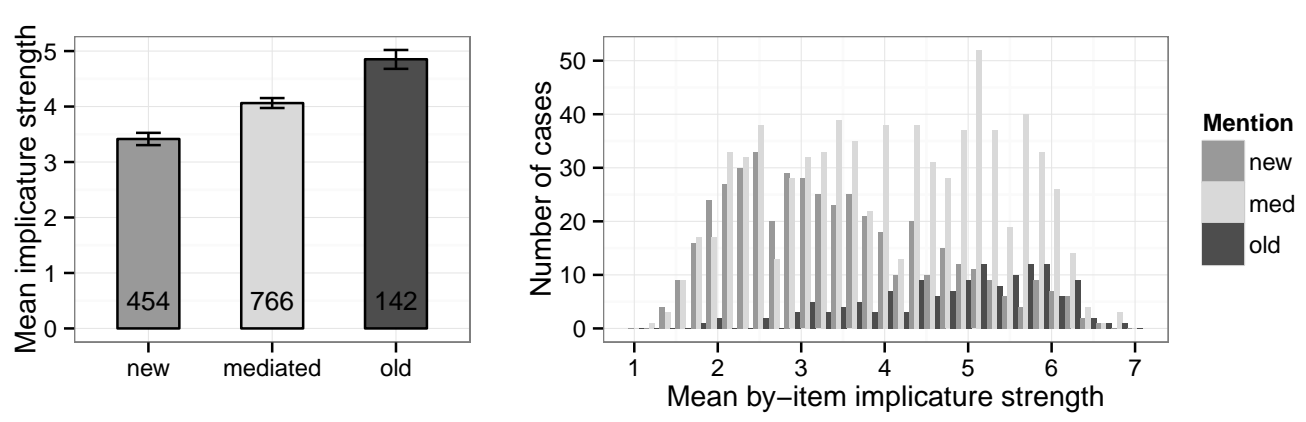

Figure 5 Mean implicature strength ratings (left) and distribution of mean by-item ratings (right) for new, mediated, and old embedded NP referents.

main effects of each of the three factors before turning to the interactions in Section 2.3.3.

Linguistic mention Nouns in the Switchboard corpus are annotated for whether they are old (previously mentioned), new (not previously mentioned), or mediated (not previously mentioned but contextually inferable) (Nissim et al. 2004). In the some-database, there were 142 old, 767 mediated, and 454 new cases. Figure 5 shows mean strength ratings for different mention categories. There is a clear gradient increase in implicature strength with increasing discourse accessibility.

For ease of analysis, old and mediated head nouns were collapsed into one category. ${ }^{28}$ As predicted, implicature ratings were higher for old than new NPs $(\beta=0.31, S E=0.07, t=4.4, p<.0001)$.

One surprising finding is that there were many new NPs that nevertheless received high implicature ratings. I discuss this further in Section 2.3.3.

Subjecthood In the Switchboard corpus, NPs are annotated for whether they are sentential subjects as in (35) or in topicalized constructions like left-dislocations as in (36).

(35) Some people are motorboaters, you know, which I think is fine. 5.5

(36) Some of those people, they don't deserve to be let loose.

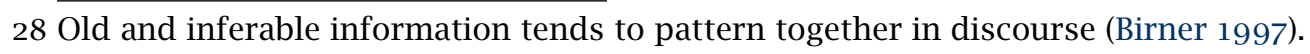



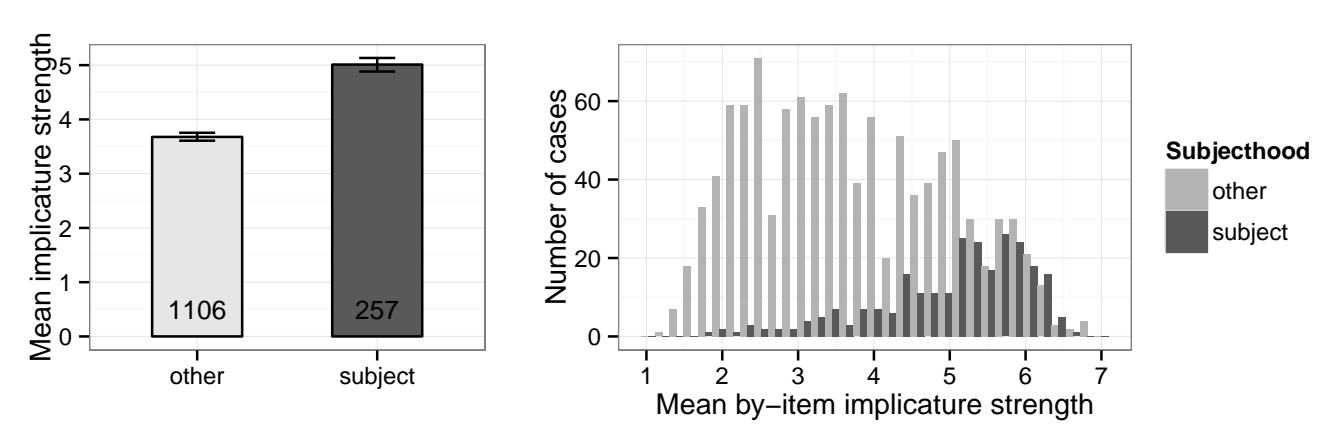

Figure 6 Mean implicature strength ratings (left) and distribution of mean by-item ratings (right) for other and subject some-NPs.

These some-NPs stand in contrast to some-NPs that occur in other positions, for instance as direct objects or in prepositional adjuncts as in (37) and (38).

(37) I've heard some horrible, horrible stories about high school teachers.

(38) We actually do some work with some people down at Georgia Tech.

Because there were only 19 cases of topicalized NPs, these were collapsed into the subject NP category. There were thus 257 subject and 1106 other NPs in the some-database. Figure 6 shows mean implicature strength ratings for subject versus other NPs: subject some-NPs give rise to stronger implicatures than other NPs $(\beta=0.41, S E=0.10, t=4.2, p<.0001)$.

Modification Finally, each case in the database was coded as either modified or unmodified, depending on whether or not the embedded NP contained pre- or post-nominal modification. For example, the examples in (39) and (40) both fell into the modified category, while the case in (41) was classified as unmodified.

(39) And then I've seen some of the Star Trek movies.

(40) We're a little farther removed from like Dallas and some of the areas where they probably have more of the homeless and that type of thing.

(41) We had some friends over as recently as Saturday night. 

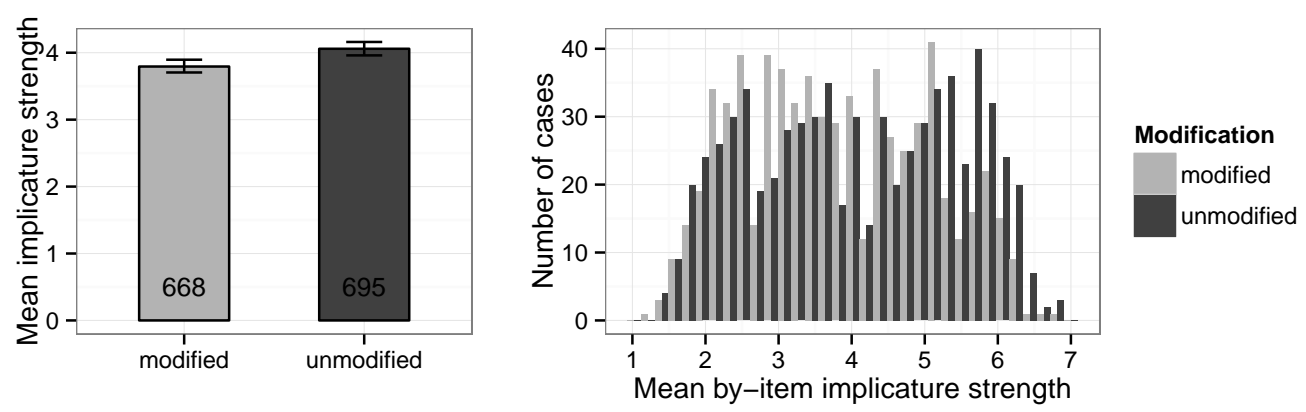

Figure 7 Mean implicature strength ratings (left) and distribution of mean by-item ratings (right) for modified and unmodified some-NPs.

This coding resulted in 667 modified and 696 unmodified cases. In addition, partitive cases with possessive embedded determiners were categorized as modified because in those cases, the determiner provided additional information about the relation between the head noun of the embedded NP and already discourse accessible entities, as in (42) where the possessive provides a link between relatives (new) and the speaker's family (old). There were 12 of these cases in the database overall.

(42) Christmas time, uh, some of our relatives would come up from $\mathrm{Al}$ abama.

Figure 7 shows mean implicature strength ratings for modified and unmodified some-NPs. Somewhat surprisingly, unmodified NPs received higher ratings than modified NPs $(\beta=0.12, S E=0.06, t=2.0, p<.05)$. This is due to an interaction with linguistic mention which is discussed in the next section.

Interactions between discourse accessibility factors The model coefficients for the two-way and three-way interactions between discourse accessibility predictors are shown in Table 3. Only the interaction between linguistic mention and modification reached significance. In addition, both the interaction between modification and subjecthood as well as the threeway interaction were trending towards significance. The three-way interaction is visualized in Figure 8. Simple slopes analysis revealed that the two-way interaction between linguistic mention and subjecthood trended towards significance for unmodified, but not for modified NPs; for modified NPs, 
Distribution of scalar implicatures

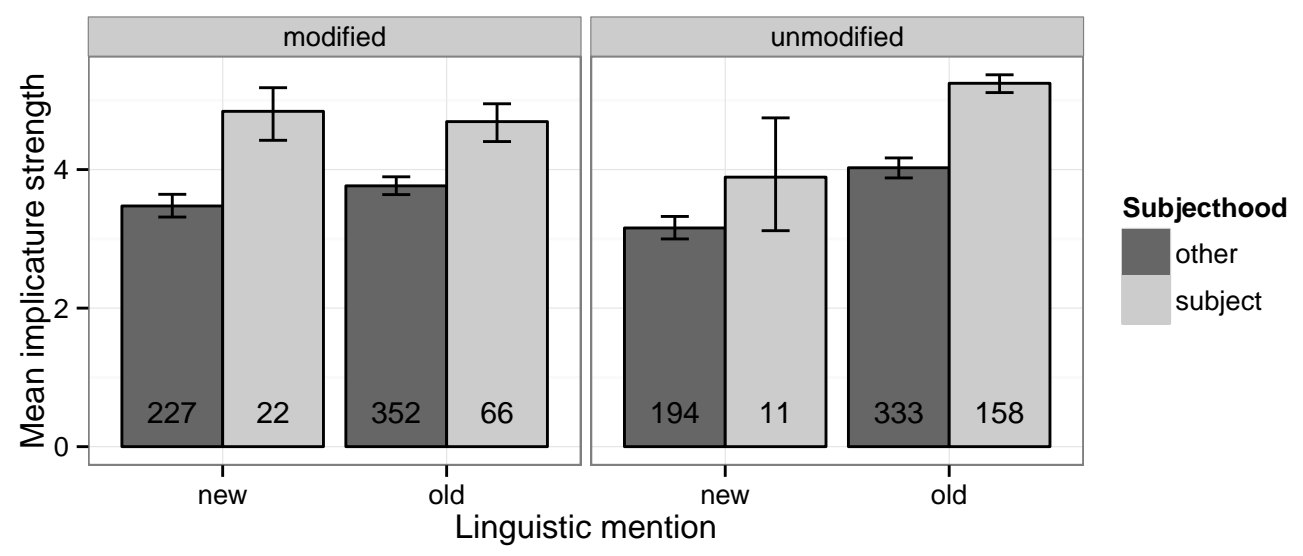

Figure 8 Mean implicature strength ratings by linguistic mention (old/new embedded NP referent), subjecthood (subject/other some-NP), and modification (modified/unmodified embedded NP). ${ }^{29}$

there was only a clear main effect of subjecthood, such that modified NPs in subject position received higher implicature ratings than modified NPs in other positions. For unmodified NPs, there was also a trending interaction such that both old and subject NPs received higher ratings, but the difference between subject and other NPs was greater for old than for new NPs.

This suggests a role for discourse accessibility that is intermediate between the two roles sketched in Section 2.3.3. As a reminder, the options were:

29 As a side note, a $\chi^{2}$ test over the linguistic mention $\times$ subjecthood contingency table replicates the well-documented tendency for subjects to favor old over new information (33 new subjects versus 180 old subjects, $\left.\chi^{2}(1)=58.73, p<.0001\right)$.

\begin{tabular}{lcccc}
\hline Predictor & $\beta$ & $S E$ & $t$ & $p$ \\
\hline Subjecthood:Mention & 0.11 & 0.21 & 0.8 & $<.43$ \\
Modification:Mention & 0.34 & 0.13 & 2.6 & $<.01$ \\
Modification:Subjecthood & 0.27 & 0.17 & 1.6 & $<.12$ \\
Modification:Subjecthood:Mention & 0.61 & 0.42 & 1.4 & $<.16$
\end{tabular}

Table 3 Model coefficients for interactions of discourse accessibility predictors. Significant effects bolded, trending effects italicized. 
i. Discourse accessibility has a gradient effect on implicature strength: the more accessible, the greater the implicature strength.

ii. Discourse accessibility has a categorical effect on implicature strength: if at least one marker of discourse accessibility is present, implicature ratings should be high, and low otherwise.

The results suggest that subjecthood has a special status: subject some-NPs boost implicature strength, no matter the presence of other discourse accessibility markers, indicating an additive effect of subjecthood, in turn supporting a gradient view of discourse accessibility. The more discourse-accessible the some-NP, the stronger the implicature. In contrast, previous mention affects implicature strength for unmodified (less discourse-accessible) NPs, but not for modified (more discourse-accessible) NPs. For cases where discourse accessibility is guaranteed (or at least increased) through modification, mention does not add an extra boost. For unmodified NPs with reduced discourse accessibility, previous mention does provide a boost. That is, there is evidence for both categorical and gradient effects of discourse accessibility on implicature strength.

\subsection{Model evaluation}

In Section 2.3 I reported and discussed the effect of multiple contextual features on scalar implicature strength. In particular, partitivity, determiner strength, and three markers of discourse accessibility (and some of their interactions) all affected implicature strength. Some readers may wonder at this point whether all of the predictors in the model are necessary, and how well the model captures the data. The first question is easy to answer: the linear regression model used guarantees that each of the significant predictors (including interaction terms) has an independent effect on implicature strength.

As for the second question, we can inspect two different measures of model quality. The first is the Bayesian Information Criterion (BIC, Schwarz 1978), a measure of model quality that takes into account the likelihood of the data, given the model. It includes a penalty for added parameters. Models with lower BIC values are preferred over models with higher BIC values. We can thus compare the final model both to a basic model that includes only by-participant random intercepts (i.e., a model that only captures baseline participant variability) and to an intermediate one that additionally contains 
Distribution of scalar implicatures

\begin{tabular}{lrrr} 
& Basic model & Intermediate model & Final model \\
\hline Marginal $R^{2}$ & 0.00 & 0.16 & 0.14 \\
Conditional $R^{2}$ & 0.09 & 0.27 & 0.46
\end{tabular}

Table 4 Proportion of variance explained by the three models.

the fixed effects of interest but does not include by-item intercepts or byparticipant random slopes for each fixed effect (i.e., a model that captures neither the way in which participants may differ in how strongly their responses are affected by each fixed effect, nor the baseline variability between items that has nothing to do with the fixed effects of interest). BIC values for the basic, intermediate, and final model are 58,453, 55,938, and 54,016, respectively. Model comparison reveals that the final model is a vast improvement over the intermediate model $\left(\chi^{2}(7)=1976, p<.0001\right)$, which in turn is a vast improvement over the basic model $\left(\chi^{2}(11)=2663, p<.0001\right)$.

A different, more intuitive way of evaluating the model is to compare the empirical data to the values predicted by each of the three (basic, intermediate, and final) models. This is visualized in Figure 9. The first observation is that the final model provides an almost perfect fit to the data $(r=.99)$, while the intermediate model $(r=.66)$ at least predicts a much wider range of values than the basic model $(r=.16)$ that only accounts for participant variability. This demonstrates

i. that participant variability is lower than the variability due to the contextual factors of interest, and

ii. that there is substantial by-item variability.

Another way to illustrate point (ii) is by comparing conditional $R^{2}$ values for generalized mixed-effects models obtained using the MuMIn package in $\mathrm{R}$ (Barton 2014). $R^{2}$ is a popular way of assessing model fit and has recently been extended to mixed models (Nakagawa \& Schielzeth 2013, Johnson 2014). Marginal $R^{2}$ represents the proportion of variance explained by fixed effects of interest, while conditional $R^{2}$ represents the proportion of variance explained by the whole model, including random effects. Adding by-item intercepts almost doubles the variance explained, as shown in Table 4, further confirming a large degree of by-item variability in implicature strength.

I briefly discuss potential sources of this item variability, some of which were already touched upon in Section 2.2.1. One source of variability may be 

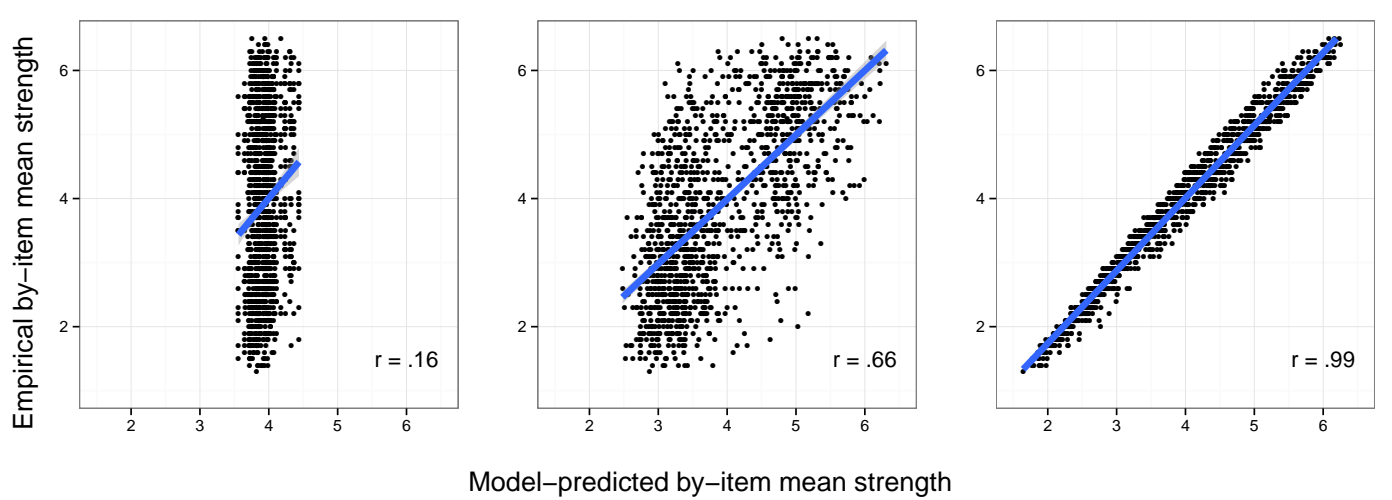

Model-predicted by-item mean strength

Figure 9 Scatterplot of empirical versus predicted mean by-item strength ratings for basic model (left panel, only by-participant random intercepts), intermediate model (center panel, additionally fixed effects of interest), and final model (right panel, additionally byitem random intercepts and by-participant random slopes for fixed effects). Blue line indicates best linear fit.

the sensitivity of scalar implicatures to embeddings within polarity affecting contexts. I have argued in Section 2.3.2 that due to the very rare occurrence of such contexts at least in the dataset reported here, this factor may play a much smaller role than assumed by some (Chierchia, Fox \& Spector 2008). More likely causes of residual variability are

i. the degree of uncertainty that hearers believe speakers to have about the truth of the stronger alternative,

ii. hearers' perceived relevance of the stronger alternative to a contextual QUD, and

iii. the prior probability of the stronger alternative being true,

all of which have been argued to play a role in deriving scalar implicatures (Grice 1975, Gazdar 1979, Horn 1989, Matsumoto 1995, Sauerland 2004, Franke 2009, Geurts 2010, Zondervan 2010, Bergen \& Grodner 2012, Russell 2012, Breheny, Ferguson \& Katsos 2013, Degen 2013, Goodman \& Stuhlmüller 2013). 
Distribution of scalar implicatures

Speaker uncertainty about the truth of the stronger alternative As has been noted, by default only a weak implicature to the effect that the speaker does not believe that the stronger alternative holds arises upon observing a scalar item like some (Gazdar 1979, Horn 1989). To get the stronger implicature that the speaker believes that the stronger alternative does not hold, the hearer must make the assumption that the speaker is knowledgeable with respect to the truth of the stronger alternative (Sauerland 2004). The local insertion of but not all into the target utterance may have shifted participants' estimates of the knowledge state that a speaker must have been in in order to produce the modified utterance, thus leading to a greater perceived dissimilarity between target and comparison utterance.

Relevance of the stronger alternative to a contextual QUD Locally inserting but not all may have shifted the salient QUD that the utterance is interpreted relative to, as discussed in Section 2.2.2. This may have also lowered participants' perceived similarity between target and comparison utterance.

Prior probability of the stronger alternative being true In some cases world knowledge about the relative probability of the stronger alternative being false, independent of any considerations of likely speaker knowledge or the QUD, may have guided participants' judgments. The following are cases that received strong implicature strength ratings despite the model predicting low ratings. The first value is the model's predicted rating, the second value the actual mean item rating.

(43) There are some Kurds living in Iran. $\quad 3.4$ / 5.0

(44) And it's a brick house, with, uh, some wood. $\quad 3.3 / 5.2$

It is clear that no inference about speaker intentions is necessary in order to express a judgment that not all Kurds live in Iran and that a brick house is not embellished with all the wood in the world.

All three of these factors are likely contributing to participants' final strength ratings in complex ways (see also Russell 2012 for a comprehensive discussion of how prior beliefs, relevance of alternatives, and other factors are expected to interact in giving rise to perceived implicature strength). Including estimates of these three factors for each of the items in the dataset 
will likely improve model fit. How these factors interact with the the contextual factors discussed in Section 2.3 is an interesting empirical question that will shed light on the relation between surface features (e.g. the partitive), semantic features of surface forms (e.g. discourse accessibility), world knowledge (e.g. prior beliefs about likely states of the world), and top-down expectations about language use (e.g. the relevance of an utterance and its alternatives to a contextual QUD), in scalar implicature computation.

\section{General discussion}

Lexicalized scalar implicatures have long been classified as GCIs (Horn 1989, Levinson 2000). The main grounds for this classification has been individual researchers' intuitions regarding the relative regularity and context independence with which scalar implicatures arise compared to more contextdependent PCIs. This paper constitutes an attempt to rigorously test the main assumption underlying the classification of scalar implicatures as GCIs, an assumption I termed the Homogeneity Assumption and spelled out in terms of two sub-assumptions: that of STRENGTH INVARIANCE (that scalar implicatures display no or little variability in the degree to which they arise) and that of CONTEXT INDEPENDENCE (that they arise independently of context).

In Section 2 I reported a test of the Homogeneity Assumption for the $\langle$ all, some $\rangle$ scale, the most clearly lexicalized of scales, in which a large number (243) of linguistically untrained language users' implicature strength judgments were collected for 1363 naturally occurring utterances containing some. This allowed for separate tests of STRENGTH INVARIANCE and CONTEXT INDEPENDENCE. First, the overall variation in participants' judgments was analyzed. In a second step, the effect of three features of context (or, from the hearer's perspective, contextual cues to the speaker's intention) on implicature strength was analyzed.

In the following I summarize the main experimental results and discuss the implications of these results for (a) the status of scalar implicatures as GCIs, (b) processing theories that rely on scalar implicatures constituting a homogeneous class of implicatures, and (c) the status of the GCI-PCI distinction itself. 
Distribution of scalar implicatures

\subsection{Summary and discussion of experimental results}

The two main results reported in this paper are (a) that scalar implicatures from some to not all vary much more than expected under the Homogeneity Assumption and (b) that implicature strength is probabilistically modulated by various features of context. In particular, implicature strength (or the degree to which a speaker is taken to implicate the negation of the stronger alternative) is greater on average when some occurs with the partitive, when its use is relatively strong, and when the embedded NP referent is relatively discourse accessible (i.e., when it has been previously mentioned or is contextually inferable, when the some-NP is in subject position, or when the embedded head noun is modified).

These results suggest that the Homogeneity Assumption, at least for scalar implicatures from some to not all, is not warranted. Not only is implicature strength highly variable between different occurrences of some (pace STRENGTH INVARIANCE), it is also systematically dependent on context (pace CONTEXT INDEPENDENCE). This result undermines the assumption made in the previous literature, which overwhelmingly treats lexicalized scalar implicatures as highly regularized, context-independent inferences (Horn 1972, Levinson 2000, Huang \& Snedeker 2009). ${ }^{30}$ It will therefore be crucial for follow-up work to establish the robustness and generalizability of the result by testing the Homogeneity Assumption both for different scales and for different dependent measures. ${ }^{31}$ It is possible that implicatures using the $\langle$ all, some $\rangle$ scale just happen to display more variation and context-dependence than expected, and other scales may indeed display the behavior expected under the Homogeneity Assumption. This is unlikely, given the status of the $\langle$ all, some $\rangle$ scale as the paradigmatic example of a lexicalized scale, which suggests that any other scale should display more, rather than less, context-dependence. But this is an empirical question that can and should be answered by digging into corpus and judgment data which are increasingly becoming available.

An additional important area for future work arises from the observation that, while the model predictions are reasonably correlated with participants' actual ratings, there is still substantial residual variability in ratings that the

30 But see Ariel 2004 for evidence that the not all implicature is similarly infrequent for most. 31 First attempts at establishing implicature strength for different scales and for different implicature types have been made by van Tiel et al. (2014) and Doran et al. (2012); they find a large degree of variability. However, in-depth studies into the context-dependence of these various implicatures remain to be conducted. 
model does not capture. As discussed in Section 2.4, this variability is likely due in large part to factors not presently included in the model, including (a) the degree of uncertainty that hearers believe speakers to have about the truth of the stronger alternative, (b) hearers' perceived relevance of the stronger alternative to a contextual QUD, and (c) the prior probability of the stronger alternative being true. Future work should attempt to estimate these quantities and integrate them into the model.

\subsection{Implications}

The results reported in this paper have implications both for theories of how scalar implicatures arise and for theories of how they are processed, which are discussed in the following.

\subsubsection{Scalar implicature and the GCI-PCI distinction}

The variability in strength and the context dependence exhibited by scalar implicatures from some to not all are at odds with the status of these implicatures as GCIs. Let's reconsider Grice's (1975) formulation of what makes a GCI: "the use of a certain form of words in an utterance would normally (in the ABSENCE of special circumstances) carry such-and-such an implicature or type of implicature". The results reported in this paper clearly indicate that an utterance of some cannot be said to normally carry a not all implicature. But maybe the parenthetical in the absence of special circumstances can help? Others, such as Horn (1984), have also remarked that a GCI should go through in unmarked contexts. So perhaps the test of the Homogeneity Assumption was unfair because it did not exclude marked contexts.

There are multiple reasons why this is not a satisfying objection. First, it is not clear what would constitute a marked context. The presence of at least one of the features that lower implicature strength, identified in Section 2.3 ? The presence of all strength-lowering features? Something in between? Excluding cases according to any of these criteria will increase implicature strength - this follows from the statistical analyses reported above. However, (a) implicature strength in the remaining cases will nevertheless not be maximal and (b) the remaining cases will retain variability in strength; that is, STRENGTH INVARIANCE will remain violated. Moreover, trying to save the GCI status by pointing to the markedness of some of the contexts included in the analysis ignores the gradient and systematic dependence of implicature 
strength on context. This context dependence is arguably a more interesting finding than variation in implicature strength; it suggests that scalar implicatures from some to not all are much more PCI-like than previously suspected.

But if some-not-all implicatures are more like PCIs than like GCIs, this does not bode well for the GCI-PCI distinction. Others have previously questioned the usefulness of the distinction or argued that it is a matter of degree rather than type (Hirschberg 1985, Wilson \& Sperber 1995, Carston 1998, van Rooij 2003). The results reported in this paper provide further evidence that, rather than conceiving of form-to-inference mapping as categorically context-dependent or context-independent, different implicature types may be more or less context-dependent. This paper has shown that some-not-all implicatures, the traditionally most context-independent of implicatures, are nevertheless systematically context-dependent. Of course there are many implicatures that are more context-dependent; but if we (cautiously) take somenot-all implicatures to provide a lower bound on the context-dependence of implicatures, categorical context-dependence cannot be used as a diagnostic of whether a particular implicature should be considered a GCI or a PCI. But context-dependence has been the diagnostic for distinguishing GCIs and PCIs. Discarding it leaves the GCI-PCI distinction with no other independent diagnostic, rendering it meaningless.

What might be an alternative view of the role of context-dependence in conversational implicatures? We would like to avoid simply saying that all implicatures are context-dependent and leaving it at that. Under the probabilistic pragmatics view sketched in Section 1.2, different implicature types fall onto a continuum of context-dependence. The challenge now lies in quantifying the degree of context-dependence for different implicature types. This paper provides an example of how, with the help of corpora of spontaneous speech as well as the web-based collection of regular language users' interpretation of utterances, one can begin to probe implicatures' degree of context-dependence. While a precise suggestion for how to quantify context-dependence in the general case lies outside the scope of this paper, the work reported here opens an exciting avenue for the development of data-driven estimates of the degree and type of context-dependence exhibited by different implicature types. 


\subsubsection{Processing theories of scalar implicature}

Overturning the Homogeneity Assumption also has consequences for theories of how scalar implicatures are processed. In Section 1.1.1 I introduced two such theories: the Default model (Levinson 2000) and the Literal-First hypothesis (Huang \& Snedeker 2009). I discuss both in turn.

The Default model The Default model assumes that the process of computing lexicalized implicatures does not incur a processing cost; only their cancellation does (Levinson 2000). Levinson sees this as a solution to the articulatory bottleneck problem: the question of how it is that communication can proceed as rapidly as it does, assuming that integration of contextual information is effortful and time-consuming. The crucial assumption that warrants this solution is precisely the Homogeneity Assumption - only if scalar implicatures from some to not all are context-independent does removing a processing cost from their computation allow for an overall processing speedup.

The results reported here thus undermine the core assumption of the Default model. ${ }^{32}$ But what would constitute an alternative solution to the articulatory bottleneck problem?

Under the probabilistic pragmatics approach, the assumption that the integration of contextual information is generally cognitively costly is relaxed. Hearers are assumed to have acquired rich, probabilistic knowledge of the contexts in which speakers intend to communicate the negation of the stronger alternative. When observing an utterance with a scalar item, hearers can then use the available contextual information to infer the speaker's intention. If contextual support for the implicature is strong, it should be computed more rapidly than if contextual support is weak. A thorough test of this prediction remains to be conducted. A further interesting question is how hearers learn to track the right kinds of contextual features.

The Literal-First processing hypothesis The Literal-First hypothesis is the hypothesis that the lower-bound interpretation of an utterance with a scalar item is computed before the pragmatically enriched upper-bound interpretation. Evidence for this staged process would provide evidence for a clear

32 There is also ample evidence from the processing literature that scalar implicatures are not computed by default in the general case (Bott \& Noveck 2004, Huang \& Snedeker 2009, 2011, but see Grodner, Klein, et al. 2010, Breheny, Ferguson \& Katsos 2013). 
distinction between semantics and pragmatics, and more importantly, for a modular view in which the pragmatics operates on the semantics. The modular view is of course widespread in linguistics - literal meanings of expressions are taken to be basic and can be pragmatically enriched. However, it is not clear that this distinction is psychologically meaningful.

The Literal-First hypothesis is associated with two key predictions: that the pragmatic upper-bound interpretation of some should be derived more slowly (a) than the literal lower-bound interpretation and (b) than other literal controls (e.g., utterances in which some is replaced by all).

The bulk of the empirical findings - from response times (Bott \& Noveck 2004, Neys \& Schaeken 2007, Bott, Bailey \& Grodner 2012), reading times (Breheny, Katsos \& Williams 2006), mouse movements (Tomlinson, Bailey \& Bott 2013), and eye movements (Huang \& Snedeker 2009, 2011) - points towards implicatures being costly, in support of the Literal-First hypothesis. However, making the Homogeneity Assumption is crucial to the interpretation of the "costly implicature" results as reflecting a literal-first process, in the following way. If the Homogeneity Assumption is not warranted, an alternative interpretation of the "costly implicature" results is at least as plausible as a literal-first processing mechanism: this alternative is that the observed delays are due to the low prior support for an implicature. To see why this should be so, a brief foray into frequency and predictability effects in other areas of language processing is required.

From a vast body of literature on frequency and predictability effects in other domains of language processing, it is well-known that more frequent or predictable words or structures are processed more quickly than less frequent or predictable words or structures. For example, more frequent words are recognized more quickly and more accurately than less frequent words (Marslen-Wilson 1987, Seidenberg \& McClelland 1990, Dahan, Magnuson \& Tanenhaus 2001). Similarly, more frequent and predictable words and structures are read more rapidly than less frequent and predictable ones (Ehrlich \& Rayner 1981, Hale 2001, Mcdonald \& Shillcock 2003, Levy 2008).

If predictability affects pragmatic processing just as it does lexical, phonological, or syntactic processing, what are the predictions for scalar implicature processing? Under the Homogeneity Assumption, implicature strength is predicted to be high and context-independent. In other words, scalar implicatures are highly predictable compared to literal interpretations of utterances with scalar items. Thus, the pragmatic interpretation of scalar expressions should be processed more rapidly than the literal one, unless literal informa- 
tion is privileged in processing. Indeed, this is exactly the argument of the Literal-First hypothesis. However, if the Homogeneity Assumption does not hold - that is, if scalar implicatures are in fact not predictable from observing a scalar expression alone - then the pragmatic interpretation should not be arrived at rapidly. And in fact, if the literal interpretation is generally more predictable than the pragmatic interpretation, then scalar implicatures are predicted to be delayed compared to literal content. But this is exactly the pattern predicted by the Literal-First hypothesis.

Thus, if the Homogeneity Assumption is not warranted, there are two alternative explanations for "costly implicature" effects: (a) the Literal-First hypothesis, and (b) the hypothesis that the less predictable interpretation is arrived at more slowly. ${ }^{33}$ The evidence against the Homogeneity Assumption reported here thus calls into question the interpretation of "costly implicature" results as unambiguous evidence for a literal-first psychological processing mechanism.

\section{Conclusion}

The main contribution of this paper is to provide evidence against the Homogeneity Assumption, an assumption that is central to the view of lexicalized scalar implicatures as GCIs. Rather than constituting a homogeneous, contextindependent class of implicatures, the results reported in this paper suggest that the strength of scalar implicatures from some to not all is highly variable and systematically context-dependent.

This work demonstrates the feasibility of large-scale experimental studies of pragmatic phenomena in naturally occurring linguistic contexts. In an era where individual researchers' judgments about hand-selected examples are no longer the only source of linguistic data available, studies of this sort will be of utmost importance in informing pragmatic theory moving forward.

\section{A Practice contexts}

(45) Practice item supporting the implicature

Speaker A: Man, this morning I wanted to have some raspberry yogurt

33 Note that the results reported here do not allow for a clear estimate of whether the literal or the pragmatic interpretation is more predictable; they do, however, raise the possibility that the literal interpretation is more predictable than the pragmatic one, and that is all that is required for the argument. 

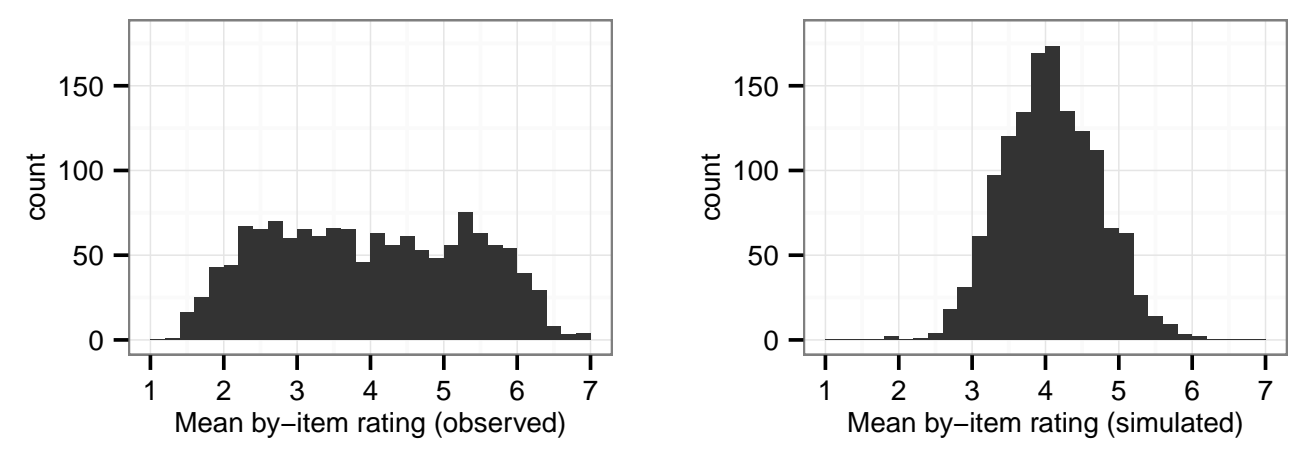

Figure 10 Distribution of observed (left panel) and simulated (right panel) mean by-item ratings.

and I checked the fridge and it was all gone.

Speaker B: Mhm.

Speaker A: Did you eat it all?

Speaker B: No I didn't! I had some of the banana yogurt.

(46) Practice item not supporting the implicature

Speaker A: Is there any food in the house?

Speaker B: Not sure. There's probably some peanuts in the pantry.

\section{B Simulation of implicature strength results under random use of scale}

To investigate the expected response distribution if participants were using the Likert scale randomly in the web-based study, and compare it to the actual distribution, a simulation was conducted. Ten (the number of judgments obtained from each participant) independent samples were drawn from a seven-point Likert scale 1363 (the number of items in the dataset) times. The resulting distribution had a mean of 4 and standard deviation 0.6 See Figure 10 for a side-by-side comparison of the observed and simulated distribution of mean by-item ratings. The distributions are very different, suggesting that participants did not use the scale randomly, but systematically. Note that the left panel is repeated from Figure 1. Differences in appearance are due to the difference in the scale on which they are plotted. 


\section{Generating strength ratings for pronominal embedded NPs}

Strength ratings for the 99 cases with pronominal embedded NP heads were not available. In order to avoid sacrificing these cases, strength ratings were generated for them in a principled way. To understand the procedure, note first that the partitive is mandatory for pronouns and demonstrative heads (see examples (47-49) together with their mean implicature rating).

(47) And some *(of) them fizzled out. $\quad 6.6$

(48) Some *(of) it sounds more like pop music. 5.9

(49) But some *(of) those are pretty big. 5.6

It is thus plausible that theses cases would receive strength ratings similar to those of the other partitive cases. Based on this assumption, ratings were generated by sampling from the strength rating distribution of the remaining 269 partitives. That is, the resulting strength distribution for pronoun/demonstrative cases was approximately the same as that of the other partitive cases. These strength ratings were used in the rest of the analysis. Excluding the pronominal head cases did not qualitatively change the results or the significance of effects.

\section{Full mixed effects linear regression model}

Table 5 contains model coefficients for the full mixed effects linear regression model predicting implicature ratings from fixed effects for cues of interest and log-transformed sentence length as well as random by-participant intercepts, by-participant slopes for all fixed effects, and by-item intercepts. All fixed effects predictors were centered before entering the analysis.

\section{References}

Ariel, Mira. 2004. Most. Language 8o(4). 658-706.

Atlas, David Jay \& Stephen C. Levinson. 1981. It-clefts, informativeness, and logical form: Radical pragmatics (revised standard version). In Peter Cole (ed.), Radical pragmatics, 1-61. New York, NY: Academic Press.

Baayen, R.H., D.J. Davidson \& D.M. Bates. 2008. Mixed-effects modeling with crossed random effects for subjects and items. Journal of Memory and Language 59(4). 390-412. http://dx.doi.org/10.1016/j.jml.2007.12.005. 
Distribution of scalar implicatures

\begin{tabular}{lrrrc}
\hline & Coef $\beta$ & SE $(\beta)$ & t & $p$ \\
\hline Intercept & 4.01 & 0.06 & 68.7 & $<. \mathbf{o o o 1}$ \\
Partitive & 0.91 & 0.09 & 9.6 & $<. \mathbf{o o o 1}$ \\
Strength & 0.50 & 0.05 & -9.5 & $<. \mathbf{o o o 1}$ \\
Linguistic mention & 0.31 & 0.07 & 4.4 & $<. \mathbf{o o o 1}$ \\
Subjecthood & 0.41 & 0.10 & 4.2 & $<. \mathbf{o o o 1}$ \\
Modification & 0.12 & 0.06 & 2.0 & $<. \mathbf{o 5}$ \\
Sentence length & 0.15 & 0.05 & 3.2 & $<. \mathbf{o 1}$ \\
Partitive:Strength & 0.39 & 0.10 & 4.1 & $<. \mathbf{o o o 1}$ \\
Linguistic mention:Subjecthood & 0.17 & 0.21 & 0.8 & $<.44$ \\
Linguistic mention:Modification & 0.34 & 0.13 & 2.6 & $<. \mathbf{0 1}$ \\
Subjecthood:Modification & 0.27 & 0.17 & 1.6 & $<.12$ \\
Linguistic mention:Subjecthood:Modification & 0.61 & 0.42 & 1.4 & $<.16$
\end{tabular}

Table 5 Model coefficients for the full model.

Barr, Dale J., Roger Levy, Christoph Scheepers \& Harry J. Tily. 2013. Random effects structure in mixed-effects models: Keep it maximal. Journal of Memory and Language 68(3). 255-278.

Barton, Kamil. 2014. Mumin: Multi-model inference. Version 1.10.5.

Barwise, Jon \& Robin Cooper. 1981. Generalized quantifiers and natural language. Linguistics and Philosophy 4(2). 159-219.

Bates, D.M., M. Maechler, B.M. Bolker \& S. Walker. 2014. Ime4: Linear mixedeffects models using Eigen and 4 . http://arxiv.org/abs/1406.5823.

Bergen, Leon \& Noah D. Goodman. 2014. The strategic use of noise in pragmatic reasoning. 36. 182-187.

Bergen, Leon \& Daniel J. Grodner. 2012. Speaker knowledge influences the comprehension of pragmatic inferences. Journal of Experimental Psychology: Learning, Memory, and Cognition 38(5). 1450-6o. http://dx.doi.org/ 10.1037/aoo27850.

Birner, Betty J. 1997. The linguistic realization of inferrable information. Language and Communication 17(2). 133-147. http://dx.doi.org/10.1126/ science.202.4366.409.

Bott, Lewis, Todd M. Bailey \& Daniel J. Grodner. 2012. Distinguishing speed from accuracy in scalar implicatures. Journal of Memory and Language 66(1). 123-142. http://dx.doi.org/10.1016/j.jml.2011.09.005. 
Bott, Lewis \& Ira Noveck. 2004. Some utterances are underinformative: The onset and time course of scalar inferences. Journal of Memory and Language 51(3). 437-457. http://dx.doi.org/10.1016/j.jml.2004.05.006.

Breheny, Richard, Heather J. Ferguson \& Napoleon Katsos. 2013. Taking the epistemic step: Toward a model of on-line access to conversational implicatures. Cognition 126(3). 423-440. http://dx.doi.org/10.1016/j. cognition.2012.11.012.

Breheny, Richard, Napoleon Katsos \& John Williams. 2006. Are generalised scalar implicatures generated by default? An on-line investigation into the role of context in generating pragmatic inferences. Cognition 100(3). 434-463. http://dx.doi.org/10.1016/j.cognition.2005.07.003.

Carlson, Greg N. 1977. A unified analysis of the English bare plural. Linguistics and Philosophy 1(3). 413-456.

Carston, Robyn. 1998. Informativeness, relevance and scalar implicature. In R. Carston \& S. Uchida (eds.), Relevance theory: Applications and implications, 179-236. Amsterdam: John Benjamins.

Chambers, Craig G., Michael K. Tanenhaus \& James S. Magnuson. 2004. Actions and affordances in syntactic ambiguity resolution. Journal of Experimental Psychology: Learning, Memory, and Cognition 30(3). 687696.

Chierchia, Gennaro. 2004. Scalar implicatures, polarity phenomena, and the syntax/pragmatics interface. In A. Belletti (ed.), Structures and beyond, vol. 3, 39-103. Oxford, UK: Oxford University Press.

Chierchia, Gennaro, Danny Fox \& Benjamin Spector. 2008. The grammatical view of scalar implicatures and the relationship between semantics and pragmatics. In Klaus von Heusinger, Claudia Maienborn \& Paul Portner (eds.), Handbook of semantics, 1-43. Berlin: Mouton de Gruyter.

Dahan, Delphine, James S. Magnuson \& Michael K. Tanenhaus. 2001. Time course of frequency effects in spoken-word recognition: Evidence from eye movements. Cognitive Psychology 42(4). 317-67. http://dx.doi.org/10. 1006/cogp.2001.0750.

Degen, Judith. 2013. Alternatives in pragmatic reasoning. Rochester, NY: University of Rochester PhD thesis.

Degen, Judith, Michael C. Franke \& Gerhard Jäger. 2013. Cost-based pragmatic inference about referential expressions. 35. 376-381.

Degen, Judith \& Noah D. Goodman. 2014. Lost your marbles? The puzzle of dependent measures in experimental pragmatics. Conference of the Cognitive Science Society (CCSS) 36. 397-402. 
Distribution of scalar implicatures

Degen, Judith \& T. Florian Jaeger. 2011. The TGrep2 Database Tools. https: //sites.google.com/site/judithdegen/publications/tdt_manual.pdf.

Degen, Judith \& Michael K. Tanenhaus. 2014. Processing scalar implicature: A constraint-based approach. Cognitive Science. http://dx.doi.org/10.1111/ cogs.12171.

Doran, Ryan, Gregory Ward, Meredith Larson, Yaron McNabb \& Rachel E. Baker. 2012. A novel experimental paradigm for distinguishing between what is said and what is implicated. Language 88. 124-154.

Ehrlich, Susan F. \& Keith Rayner. 1981. Contextual effects on word perception and eye movements during reading. Journal of Verbal Learning and Verbal Behavior 20. 641-655.

Frank, Michael C. \& Noah D. Goodman. 2012. Predicting pragmatic reasoning in language games. Science 336. 998.

Franke, Michael C. 2009. Signal to act: Game theory in pragmatics. Amsterdam: University of Amsterdam PhD thesis.

Gazdar, Gerald. 1979. Pragmatics: Implicature, presupposition, and logical form. New York, NY: Academic Press.

Geurts, Bart. 2010. Quantity implicatures. Cambridge, UK: Cambridge University Press.

Geurts, Bart \& Nausicaa Pouscoulous. 2009. Embedded implicatures?!? Semantics and Pragmatics 2. 1-34. http://dx.doi.org/10.3765/sp.2.4.

Gibson, Edward, Steve Piantadosi \& Kristina Fedorenko. 2011. Using Mechanical Turk to obtain and analyze English acceptability judgments. Language and Linguistics Compass 5(8). 509-524. http://dx.doi.org/10.1111/j.1749818X.2011.00295.X.

Godfrey, J.J, E.C. Holliman \& J. McDaniel. 1992. SwITchBOARD: A telephone speech corpus for research and development. International Conference on Acoustics, Speech and Signal Processing (ICASSP). 517-520.

Goodman, Noah D. \& Andreas Stuhlmüller. 2013. Knowledge and implicature: Modeling language understanding as social cognition. Topics in Cognitive Science 5(1). 173-84. http://dx.doi.org/10.1111/tops.12007.

Grice, Herbert Paul. 1975. Logic and conversation. Syntax and Semantics 3. Peter Cole \& Jerry L. Morgan (eds.). 41-58.

Grodner, Daniel J., Natalie M. Klein, Kathleen M. Carbary \& Michael K. Tanenhaus. 2010. "Some," and possibly all, scalar inferences are not delayed: Evidence for immediate pragmatic enrichment. Cognition 116(1). 42-55. http://dx.doi.org/10.1016/j.cognition.2010.03.014. 
Grodner, Daniel J. \& Julie C. Sedivy. 2011. The effect of speaker-specific information on pragmatic inferences. In Neal Pearlmutter \& Edward Gibson (eds.), The processing and acquisition of reference, 239-272. Cambridge, MA: MIT Press.

Hale, John. 2001. A probabilistic Earley parser as a psycholinguistic model. North American Chapter of the Association for Computational Linguistics (NAACL) 2. 159-166.

Heller, Daphna, Daniel J. Grodner \& Michael K. Tanenhaus. 2008. The role of perspective in identifying domains of reference. Cognition 108(3). 831-836. http://dx.doi.org/10.1016/j.cognition.2008.04.008.

Hirschberg, Julia. 1985. A theory of scalar implicature. Philadelphia, PA: University of Pennsylvania PhD thesis.

Horn, Laurence. 1972. On the semantic properties of the logical operators in English. Los Angeles, CA: University of California, Los Angeles $\mathrm{PhD}$ thesis.

Horn, Laurence. 1984. Toward a new taxonomy for pragmatic inference: Q-based and R-based implicature. In Deborah Schiffrin (ed.), Meaning, form, and use in context: Linguistic applications, 11-42. Washington, DC: Georgetown University Press.

Horn, Laurence. 1989. A natural history of negation. Chicago, IL: Chicago University Press.

Horn, Laurence. 1997. All John's children are as bald as the King of France: Existential import and the geometry of opposition. Chicago Linguistic Society (CLS) 33. 155-179.

Huang, Yi Ting \& Jesse Snedeker. 2009. Online interpretation of scalar quantifiers: Insight into the semantics-pragmatics interface. Cognitive Psychology 58(3). 376-415. http://dx.doi.org/10.1016/j.cogpsych.2008.09.001.

Huang, Yi Ting \& Jesse Snedeker. 2011. Logic and conversation revisited: Evidence for a division between semantic and pragmatic content in realtime language comprehension. Language and Cognitive Processes 26(8). 1161-1172.

Israel, Michael. 1999. "Some" and the pragmatics of indefinite construal. Proceedings of the Berkeley Linguistics Society 25. 169-182.

Jackendoff, Ray. 1977. X-bar syntax: A study of phrase structure. Cambridge, MA: MIT Press.

Jaeger, T. Florian. 2006. Redundancy and reduction in spontaneous speech. Stanford, CA: Stanford University PhD thesis. 
Distribution of scalar implicatures

Jaeger, T. Florian. 2008. Categorical data analysis: Away from ANOVAs (transformation or not) and towards logit mixed models. Journal of Memory and Language 59(4). 434-446. http://dx.doi.org/10.1016/j.jml.2007.11.007.

Jaynes, Edwin. 1979. Where do we stand on maximum entropy? In R. Levine \& M. Tribus (eds.), The maximum entropy formalism, 15-118. Cambridge: MIT Press.

Johnson, Paul C.D. 2014. Extension of Nakagawa \& Schielzeth's $R^{2}{ }_{G L M M}$ to random slopes models. Methods in Ecology and Evolution 5 (9). 944-946. http://dx.doi.org/10.1111/2041-210X.12225. http://doi.wiley.com/10.1111/ 2041-210X.12225.

de Jong, F.M.G. \& H.J. Verkuyl. 1985. Generalized quantifiers: The properness of their strength. In J. van Benthem \& A. ter Meulen (eds.), Generalized quantifiers: Theory and applications, 21-43. Dordrecht: Foris Publications. van Kuppevelt, J. 1996. Inferring from topics. Linguistics and Philosophy 19(4). 393-443.

Kuznetsova, Alexandra, Per Bruun Brockhoff \& Rune Haubo Bojesen Christensen. 2014. ImerTest: Tests for random and fixed effects for linear mixed effect models (lmer objects of lme4 package). R package version 2.0-11. http://CRAN.R-project.org/package=lmerTest.

Ladusaw, William A. 1982. Semantic constraints on the English partitive construction. West Coast Conference on Formal Linguistics (WCCFL) 1. D. Flickinger, M. Macken \& N. Wiegand (eds.). 231-242.

Ladusaw, William A. 1994. Thetic and categorical, stage and individual, weak and strong. Semantics and Linguistic Theory (SALT) 4. 220-229.

Levinson, Stephen C. 200o. Presumptive meanings: The theory of generalized conversational implicature. Cambridge, MA: MIT Press.

Levy, Roger. 2008. Expectation-based syntactic comprehension. Cognition 106(3). 1126-77. http://dx.doi.org/10.1016/j.cognition.2007.05.006.

Lumsden, M. 1988. Existential sentences: Their structure and meaning. London: Croom Helm.

Marcus, G.F., S. Vijayan, S. Bandi Rao \& P.M. Vishton. 1999. Rule learning by seven-month-old infants. Science 283(5398). 77-80.

Marslen-Wilson, W.D. 1987. Functional parallelism in spoken word-recognition. Cognition 25(1-2). 71-102.

Matsumoto, Yo. 1995. The conversational condition on Horn scales. Linguistics and Philosophy 18. 21-6o. 
Mcdonald, Scott A. \& Richard C. Shillcock. 2003. Eye movements reveal the on-line computation of lexical probabilities during reading. Psychological Science 14(6). 648-652. http://dx.doi.org/10.1046/j.o956-7976.2003.psci.

McNally, Louise \& Veerle Van Geenhoven. 1998. Redefining the weak/strong distinction. Paper presented at the 1997 Paris Syntax and Semantics Colloquium.

Milsark, Gary. 1974. Existential sentences in English. Cambridge, MA: MIT PhD thesis.

Milsark, Gary. 1977. Toward an explanation of certain peculiarities of the existential construction in English. Linguistic Analysis 3. 1-30.

Nakagawa, Shinichi \& Holger Schielzeth. 2013. A general and simple method for obtaining $R^{2}$ from generalized linear mixed-effects models. Methods in Ecology and Evolution 4(2). 133-142. http://dx.doi.org/10.1111/j.2041210x.2012.00261.x. http://dx.doi.org/10.1111/j.2041-210x.2012.00261.x.

Neys, Wim De \& Walter Schaeken. 2007. When people are more logical under cognitive load: Dual task impact on scalar implicature. Experimental Psychology 54(2). 128-133. http://dx.doi.org/10.1027/1618-3169.54.2.128.

Nissim, M., S. Dingare, J. Carletta \& Mark Steedman. 2004. An annotation scheme for information status in dialogue. Language Resources and Evaluation (LREC) 4.

Reed, A.M. 1991. On interpreting partitives. In D.J. Napoli \& J.A. Kegl (eds.), Bridges between psychology and linguistics: A Swarthmore festschrift for Lila Gleitman, 207-223. Hillsdale, NJ: Erlbaum.

Roberts, Craige. 2012. Information structure in discourse: Towards an integrated formal theory of pragmatics. Semantics and Pragmatics 5(6). 1-69. http://dx.doi.org/10.3765/sp.5.6.

Rohde, Douglas. 2005. TGrep2 User Manual. http://tedlab.mit.edu/dr/ Tgrep2/tgrep2.pdf.

van Rooij, Robert. 2003. Conversational implicatures and communication theory. In J. van Kuppevelt \& R. Smith (eds.), Current and new directions in discourse and dialogue, 283-303. Dordrecht: Kluwer.

Russell, Benjamin. 2012. Probabilistic reasoning and the computation of scalar implicatures. Providence, RI: Brown University PhD thesis.

Sauerland, Uli. 2004. Scalar implicatures in complex sentences. Linguistics and Philosophy 27(3). 367-391. http://dx.doi.org/10.1023/B:LING.00ooo23378. 71748.db.

Schwarz, Gideon E. 1978. Estimating the dimension of a model. Annals of Statistics 6(2). 461-464. 
Distribution of scalar implicatures

Sedivy, Julie C., Michael K. Tanenhaus, C.G. Chambers \& G.N. Carlson. 1999. Achieving incremental semantic interpretation through contextual representation. Cognition 71(2). 109-147.

Seidenberg, Mark S. \& James L. McClelland. 1990. A distributed, developmental model of word recognition and naming. Psychological Review 96(4). 523568.

Tanenhaus, Michael K., Michael J. Spivey-Knowlton, Kathleen M. Eberhard \& Julie C. Sedivy. 1995. Integration of visual and linguistic information in spoken language comprehension. Science 268. 1632-1634.

Team, R Core. 2014. R: A Language and Environment for Statistical Computing. R Foundation for Statistical Computing. Vienna, Austria. http://www.Rproject.org/.

van Tiel, Bob, Emiel van Miltenburg, Natalia Zevakhina \& Bart Geurts. 2014. Scalar diversity. Journal of Semantics. http://dx.doi.org/10.1093/jos/ ffuol7.

Tomlinson, John M., Todd M. Bailey \& Lewis Bott. 2013. Possibly all of that and then some: Scalar implicatures are understood in two steps. Journal of Memory and Language 69(1). 18-35. http://dx.doi.org/10.1016/j.jml. 2013.02.003.

Webber, Bonnie L. 1983. So what can we talk about now? In Michael Brady \& Robert Berwick (eds.), Computational models of discourse, 331-371. Cambridge, MA: MIT Press.

Wilson, Deirdre \& Dan Sperber. 1995. Relevance theory. In G. Ward \& L. Horn (eds.), Handbook of pragmatics, 607-632. Oxford, UK: Blackwell.

Zondervan, Arjen. 2010. Scalar implicatures or focus: An experimental approach. Amsterdam: University of Utrecht PhD thesis.

Judith Degen

Department of Psychology

450 Serra Mall

Stanford University

Stanford, CA 94305

jdegen@stanford.edu 